\title{
ats
}

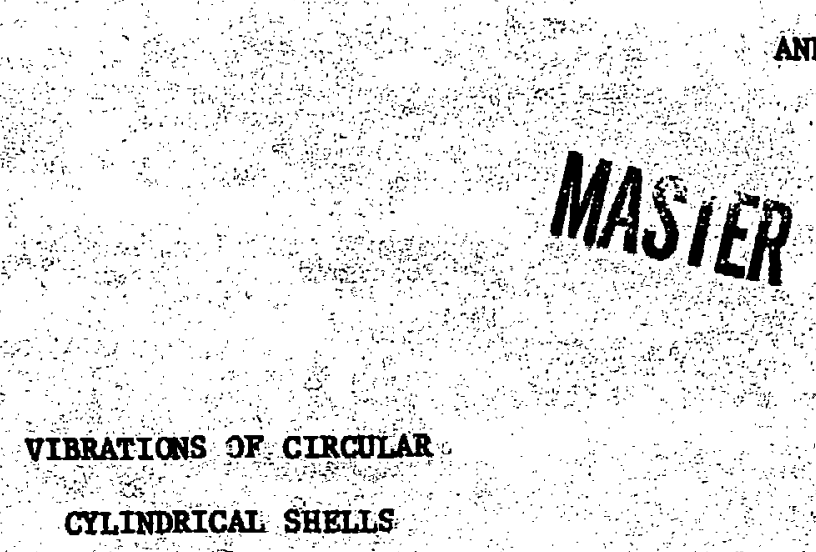

by

Ho Chung

Components Technology Dlvlsion

s

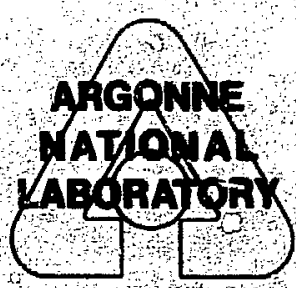

UD OLUA USERA

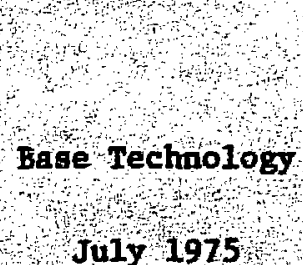

8

Ju1y 1975

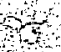

4 


\section{VIBRATIONS OF CIRCULAR}

CYLINDRICAL SHELLS

by

Ho Chung

Components Technology Division
Thls repont wat prepatel as an acsount of work

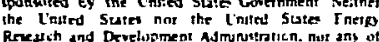
Ther ent and Develus any of thes contractorts

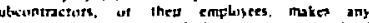

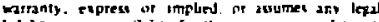

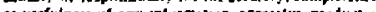

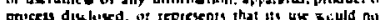

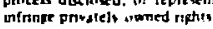

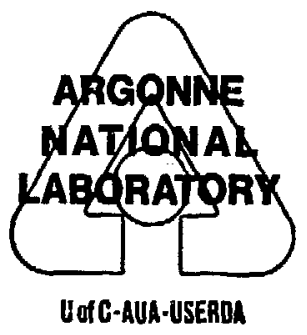

Base Technology

July 1975 
PREFACE

The work reported herein was performed as part of the base technology activity under the Flow Induced Vibration Program (189a No. CA054-A; 01d No. 02659-A) sponsored by ERDA/RRD. The overall objective of the activity is to develop new and/or improved analytical methods and guidelines for designing LMFBR components to avoid detrimental flow induced vibration.

In this report, a general analytical method is presented for evaluating the natural frequencies and mode shapes of a circular cylindrical shell with homogeneous boundary conditions of any type. The solution is obtained through a direct solution procedure using Sanders' silell equations in which the axial modal displacements are represented as simple Fourier series expressions. Stokes' transformation is used to legitimize the derivatives of the Fourier series. From the exact frequency equation obtained, the numerical results are calculated.

The method is applicable to the dynamic analysis of reactor internal and plant components that can be represented as circular cylindrical shells; the thermal liner of the Fast Test Reactor (FTR) is such a component. The thernal liner is a relatively thin shell concentric with and separated by a narrow fluid-filled gap from the main reactor vessel. The main function of the thermal liner is to shield the reactor vessel from excessive heat from the core and thereby to protect it from possible damage. The thermal liner is subjected to sodium coolant flow, and is thus susceptible to flow induced vibrations. The excitation mechanism may be associated with random pressure fluctuations, fluidelastic instabilities, or resonant vibration resulting from a coincidence between component natural frequencles and vortex-shedding or other flow-related driving frequencles. As an example of the application of the developed method, the determination of 
the free vibration modal characteristics of the thermal liner is presented and discussed. 
TABLE OF CONTENTS

$\underline{\text { Page }}$

PREFACE

TABLE OF CONTENTS

3

LIST OF FIGURES

LIST OF TABLES

NOMENCLATURE

$\begin{array}{ll}\text { ABSTRACT } & 8\end{array}$

I. INTRODUCTION 9

II. THEORETTCAL BACKGROUND 11

A. Equations of Motion 11

$\begin{array}{ll}\text { B. Modal Functions } & 13\end{array}$

C. Stokes Transformation 15

$\begin{array}{ll}\text { III. GENERAL FORMULATIONS } & 17\end{array}$

IV. FREQUENCY DETERMINANT

A. Simply-Supported Shell with No Axial Constraints at Both Ends (SNA-SNA) 23

B. Simply-Supported Shel1 (SS-SS) 24

C. Shell with Clamped Ends (C-C) 25

D. Shell with Free Ends (F-F) 25

E. Clamped-Free Shell (C-F) 26

$\begin{array}{ll}\text { V. DISCUSSIONS OF RESULTS } & 27\end{array}$

A. Convergence and Accuracy 27

B. Vibrations of Thermal Liner 30

VI. CONCLUSIONS $\quad 37$

APPENDICES

A. Boundary Conditions of Circular Cylindrical Shell 39

B. Shell Boundary Conditions to be Satisfied on Using the First (CSS) or the Seconc (SCC) Set 
TABLE OF CONTENTS (Contd.)

Paf̧e

C. Irirst Set (CSS) of Displacement Functions and their Derivatives

41

D. Symbols used in Equations (14) - (28) and Appendix E

E. Elements of Frequency Determinant Using the First Set (CSS) of Displacement Functions 


\section{LIST OF FIGURES}

No.

Title

Page

1

Cylindrical Shell with Applied Exices

\section{LIST OF TABLES}

No.

Title

$\underline{\text { Page }}$

1

Convergence of Natural Frequency Parameters, $\omega / \omega_{0}$

28

2

Comparison of Natural Frequency Parameters, $\omega / \omega_{0}$

3 Comparison of Natural Frequencies for the Thermal

Liner with Clamped-Free Boundary Conditions

Natural Frequencies of Thermal Liner for Various

Boundary Donditions

Comparisons of the Present Method with the Other

Exact Solution Method 
NOMENCLATURE
$h, R, \ell$
Thickness, radius and length of shell
k
Fourier component in axial direction
m
Number of axial mode number
n
Number of circumferential waves
u, v, w
Axial, circumferential and radial displacement
$x, \theta$
Axial, and circumferential coordinates
t
time
$v_{0}, v_{l}, w_{0}, w_{l}$ Unspecified end values
$A_{k n}, B_{k n}, C_{k n}$
D
Fourier coefficients for $u, v$ and $w$
E
Extensional rigidity, Eh/ $\left(1-v^{2}\right)$
Young's modulus
G
Shear modulus
K
Flexural rigidity, $\mathrm{Eh}^{3} / 12\left(1-\mathrm{v}^{2}\right)$
$\mathbf{M}_{\mathbf{X}}, \mathbf{M}_{\theta}, \overline{\mathrm{M}}_{\mathbf{X} \theta}$
Bending and twisting moment per unit length
$N_{x}, N_{\theta}, \bar{N}_{x \theta}$
Membrane force per unit length
$\hat{\mathbf{N}}_{\mathbf{x} \theta}$
Effective membrane shear force per unit length
$\hat{\mathrm{Q}}_{\mathrm{x}}$
Ef fective transverse shear force per unit lengih
$r^{2}$
$1 / \omega_{0}^{2}=\rho R^{2}\left(1-v^{2}\right) / E$
$\delta$
$h^{2} / 12 R^{2}$
$v$
Poisson's ratio
$\rho$
Mass density
$\psi_{u}, \psi_{v}, \psi_{w}$
Axial mode shapes corresponding to axial, circumferential,
and radial directions. respectively
$\omega$
Circular frequency of shell
$\omega_{0}$
Lowest extensional frequency of a ring in plain strain $\left(=\sqrt{E /\left\{\rho R^{2}\left(1-v^{2}\right)\right\}}\right)$
Frequency parameter $\left(=\omega^{2} / \omega_{0}^{2}\right)$ 
NOMENCLATURE (Contd.)

$$
\begin{aligned}
& (\ldots)^{\prime}=d() / d x \\
& (\ldots)_{, x}=\partial() / \partial x \\
& (\ldots)_{, \theta}=\partial() / \partial \theta \\
& (\ldots)_{, t}=\partial() / \partial t
\end{aligned}
$$




\section{VIBRATIONS OF CIRCULAR}

CYLINDRICAL SHELLS

by

Ho Chung

ABSTRACT

An exact solution method for the free vibration problem of thin circular cylindrical shells is presented. The differential equations of motion are solved directly with the use of simple Fourier series as the modal displacement functions. Stokes' transformation is exploited to obtain correct series expressions for the derivatives of the Fourier series. From this method an explicit expression of the exact frequency equation can be obtained for any kind of boundary conditions. The accuracy of the present method is checked against available data. The proposed method is then used to find the modal characteristics of the thermal liner of the Fast Test Reactor (FTR). The numerical results obtained are compared with finite element method solutions. 


\section{I . INTRODUCTION}

The vibration of thin circular cylindrical shells has been of great interest to many structural engineers ir recent years. Many investigations following the pioneering work of Arnold and Warburton $[1,2]^{*}$ have been summarized $b_{j}$ Lesssa [3]. Methods of solution for linear shell vibrations have ranged from approximate energy methods by Arnold and Warburton $\left[1,{ }^{7}\right]$ and Sharma $[4,5]$ to the exact solutions as studied by Forsberg $[6,7$, 8]. Warburton [9], Warburton and Higgs [10], and Goldman [11]. Recently, the prezent auchur used a modified Rayleigh-Ritz precedure to obtain exact soluLions [12]. In this report, the differential equations of shell are solved directly, and an exact frequency equation is obtained.

The problem is treated within the framework of linear, elastic, firstorder shell theory. Starting with Sanders' theory [13], the dependent variables are expanded in Fourier series in the circumferential direction. The problem is then considered on a mode-by-mode basis associated with the circumferential Fourier index n. In the axial direction, the variables are built up to form a set of complete orthogonal functions in the form of simple Fourier ser ies which are not required to satisfy the boundary conditions term by term. Stokes' transformation [15] is used to obtain correct series expressions for derivatives of the Fourier series. The boundary conditions which are not satisfied directly by the assumed series are enforcad. The unwanted boundary conditions, that are originally unspecified but are implied by the nature of the assumed series, are released to remain unspecified. Through this process, all the boundary conditions of the problem become satisfled by the assumed displacement function in an overall sense. It should be empnasized that the present method can solve problems

\footnotetext{
* Numbers in brackets designate References at the end of the report.
} 
with any kind of boundary conditions. The direct solution method used in this report differs from those of other contributors [6-11] in the selection of general solutions of shell equations. They chose exponential functions for the modal displacemunts along the axial direction and substituted them into the equations of motion and enforced the eight specified boundary conditions. This led to an eighth-order algebraic equation and an eighthcrder frequency determinant which are coupled together. The simultaneous solution of these two systems of equations involves extremely laborious computation [16]. In the present report, the use of simple Fourier series along the axial direction greatly simplifies the solution procedure and leads to a frequency determinant of low order ( $\leq 4)$. The numerical results from this method are checked with the available exact solutions reported by other contributors. The natural frequencies of the thermal 1iner model of the Fast Test Reactor (FTR) are calculated and discussed. In particular, the variation of natural frequencies due to the change of boundary conditions is discussed in detail. 


\section{THEORETICAI BACKGROUND}

A. Equations of Motion

First-order liner shell theories, based on Karchhoff's hypothesis, have been given by Donne11, Love, Timoshenko, Novozhilov, Flügge, Vlasov, Sanders, and others. For an excellent summary see Leissa [3]. Sanders' shel1 equations [13] are said to be "best" for the following reasons [14]: they can be written in general tensor form for arbitrary shell geometry; the principle of virtual work, the minimum energy and the reciprocal relations all apply; the stresses are described by six stress measures that satisfy the equations of equilibrium without approximation; the stress and strain measures obey the uncoupled Love constitutive relations, etc. The celebrated Fligge's equations cannot be given in general tensor form, and they do not satisfy the uncoupled constitutive laws. The present author has chosen Sanders' snell equations

$$
\begin{aligned}
& \mathrm{R}^{2} \mathrm{u}, \mathrm{xx}+\frac{1-v}{2}\left(1+\frac{\delta}{4}\right) \mathrm{u}_{, \theta \theta}+\mathrm{R}\left\{\frac{1+v}{2}-\frac{3(1-v)}{8} \delta\right\} \mathrm{v}, \mathrm{x} \theta \\
& +v^{\mathrm{Rw}}, \mathrm{x}+\frac{1-v}{2} \mathrm{R} \delta \mathrm{w}_{, \mathrm{x} \theta \theta}=\gamma^{2} \mathrm{u}_{, \mathrm{tt}} \\
& \mathrm{R}\left\{\frac{1+v}{2}-\frac{3(1-v)}{8} \delta\right\} \mathrm{u}_{, x \theta}+(1+\delta) v_{, \theta \theta}+\frac{1-v}{2} \mathrm{R}^{2}\left(1+\frac{9 \delta}{4}\right) \mathrm{v}, \mathrm{xx} \\
& -\frac{(3-v)}{2} \mathrm{R}^{2} \delta w_{, \mathrm{xx} \theta}+w_{, \partial}-\delta{ }_{, \theta \theta \theta}=\gamma^{2} \mathrm{v}, \mathrm{tt} \\
& \frac{1-v}{2} R \delta u_{, x \theta \theta}+v R u, x-\frac{3-v}{2} R^{2} \delta v, x x \theta+v, \theta+w
\end{aligned}
$$

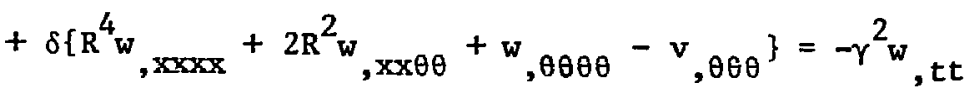

where

$$
\gamma^{2}=\rho \mathrm{R}^{2}\left(1-v^{2}\right) / \mathrm{E}, \quad \delta=\mathrm{h}^{2} / 12 \mathrm{R}^{2} .
$$

The geometry of a circular cylindrical shell is showr in Fig. 1. Also shown on the figure are the forces and moments acting on a section of the she11 parallel to the coordinate lines. 

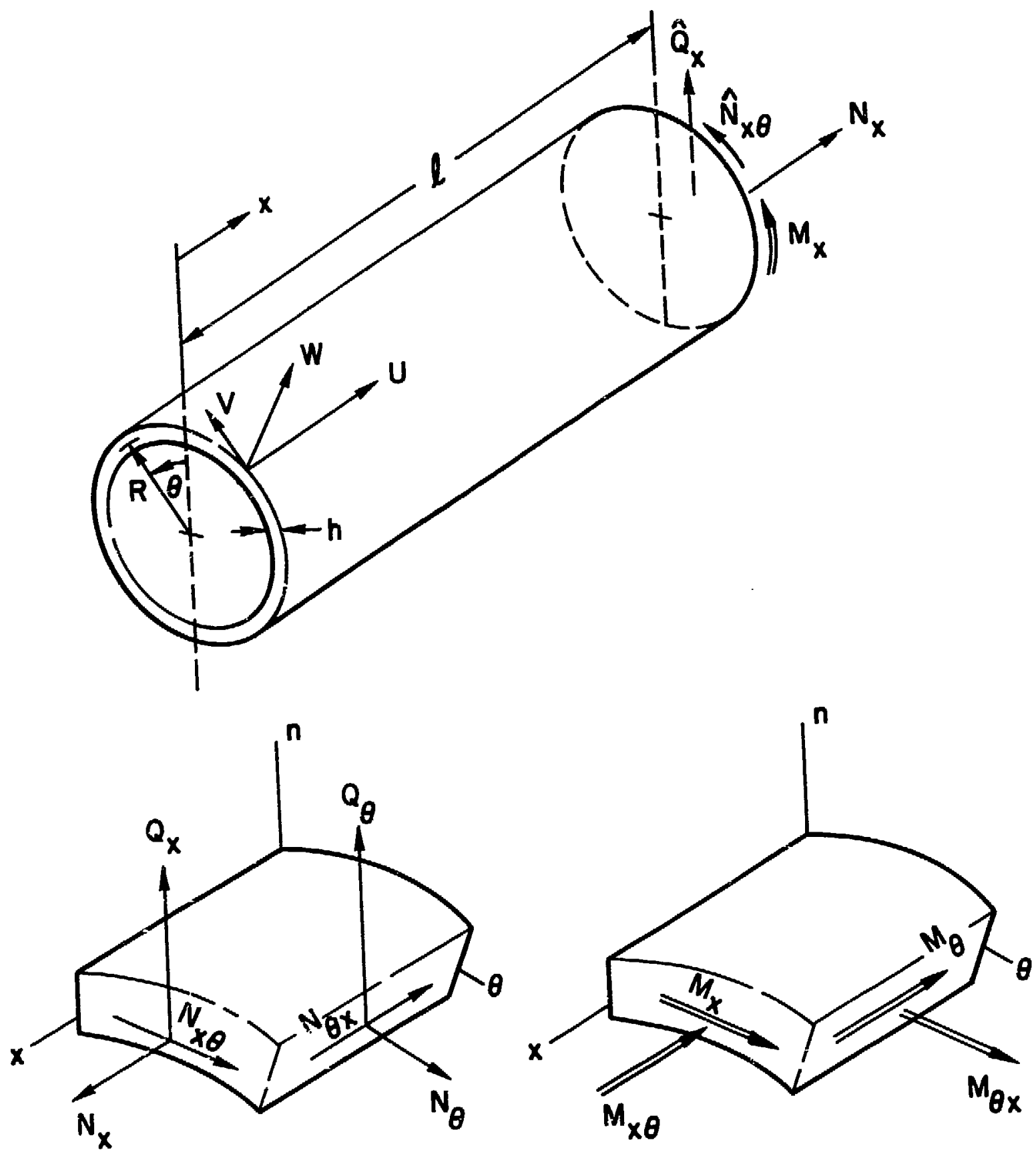

Fig. 1. Cylindrical Shell with Applied Forces 
The boundary conditions applicable to Sanders' theory reduce to prescribing the following quantities at the ends of the cylindrical shell:

$$
\left.\begin{array}{ll}
N_{x} \text { or } u, & \hat{N}_{x \theta} \text { or } v \\
\hat{Q}_{x} \text { or } w, & M_{x} \text { or } \frac{\partial w}{\partial x}
\end{array}\right\} x=0,0
$$

where

$$
\hat{\mathrm{N}}_{x \theta}=\overline{\mathrm{N}}_{x \theta}+\frac{3}{2 \mathrm{R}} \overline{\mathrm{M}}_{\mathrm{x} \theta}, \quad \hat{\mathrm{Q}}_{\mathrm{x}}=\frac{\partial \mathrm{M}}{\partial \mathrm{x}}+\frac{2}{\mathrm{R}} \frac{\partial \overline{\mathrm{M}} \mathrm{x} \theta}{\partial \theta}
$$

The "effective" forces per unit length $\hat{\mathrm{N}}_{x}$ and $\hat{Q}_{x}$ are shown in Fig. 1 . The force quantities on the left in $(2)^{*}$ are associated with the natural boundary conditions while the displacement quantities on the right are associated with the geometric boundary conditions. Further information on these relationships may be found in References [12] and [13]. It will prove to be useful to have the relationships between boundary forces and displacements explicitly written out,

$$
\begin{aligned}
& \mathrm{N}_{\mathrm{x}}=\mathrm{D}\left[\mathrm{u}_{, \mathrm{x}}+\frac{\nu}{\mathrm{R}} \mathrm{v}_{, \theta}+\frac{v}{\mathrm{R}} \mathrm{w}\right] \\
& \hat{\mathrm{N}}_{\mathrm{x} \theta}=\frac{\mathrm{D}(1-v)}{2}\left[\frac{1}{\mathrm{R}}\left(1-\frac{3}{4} \delta\right) \mathrm{u}_{, \theta}+\left(1+\frac{9}{4} \delta\right) \mathrm{v}_{, \mathrm{x}}-3 \delta \mathrm{w}, \mathrm{x \theta}\right] \\
& \hat{Q}_{x}=k\left[-\frac{(1-v)}{2 R^{3}} u, \theta \theta+\frac{3-v}{2 R^{2}},, x \theta-\frac{2-v}{R^{2}} w, x \theta \theta-w, x x x\right] \\
& M_{X}=K\left[\frac{\nu}{R^{2}}(v, \theta-w, \theta \theta)-w, x x\right]
\end{aligned}
$$

where $D=E h /\left(1-v^{2}\right), \quad K=E h^{3} / 12\left(1-v^{2}\right)$.

\section{B. Modal Functions}

The modes of vibration are characterized by the number, $n$, of circumferential waves and the number, $m$, of axial waves. For each $n, m$ combination three natural frequencies exist $[1,2]$. The essential differeire between these three vibration forms are the relative amplitudes in the axial, circumferential and radial directions. It can be shown $[1,2]$ that for any $n$, a

* Numbers in parentheses designate the equation numbers in the report. 
general relation for the displacements in any mode may be written in the following form:

$$
\begin{aligned}
& u(x, \theta, t)=\psi_{u}(x) \cos n \theta \sin \omega t \\
& v(x, \theta, t)=\psi_{v}(x) \sin n \theta \sin \omega t \\
& w(x, \theta, t)=\psi_{w}(x) \cos n \theta \sin \omega t
\end{aligned}
$$

where $\psi_{\mathrm{u}}, \psi_{\mathrm{v}}, \psi_{\mathrm{w}}$ are mode functions corresponding to axial, tangential and radial displacements, respectively. The cruclal part of the analysis involves choosing appropriate series forms for these mode functions. The series should be simple in form and at the same time preserve orthogonality properties. It is not necessary that the series satisfy any particular boundary conditions since we are dealing with a general solution at this point.

There are two convenient sets of Fourier series that meet al1 these requirements for the mode functions along the axial direction. The first set, designated "css," can be written in the form

$$
\begin{aligned}
& \psi_{u}(x)=A_{o n}+\sum_{k=1}^{\infty} A_{k n} \cos \frac{k \pi x}{\ell} \\
& \psi_{v}(x)=\sum_{k=1}^{\infty} B_{k n} \sin \frac{k \pi x}{\ell} \\
& \psi_{w}(x)=\sum_{k=1}^{\infty} C_{k n} \sin \frac{k \pi x}{\ell}
\end{aligned}
$$

while the second set, designated "SCC," can be written as

$$
\begin{aligned}
& \psi_{\mathrm{u}}(\mathrm{x})=\sum_{\mathrm{k}=1}^{\infty} A_{\mathrm{kn}} \sin \frac{\mathrm{k \pi x}}{\ell} \\
& \psi_{\mathrm{v}}(\mathrm{x})=\mathrm{B}_{\mathrm{on}}+\sum_{\mathrm{k}=1}^{\infty} \mathrm{B}_{\mathrm{kn}} \cos \frac{\mathrm{k \pi x}}{\ell} \\
& \psi_{\mathrm{w}}(\mathrm{x})=\mathrm{C}_{\text {on }}+\sum_{\mathrm{k}=1}^{\infty} C_{k n} \cos \frac{\mathrm{k} \pi \mathrm{x}}{\ell}
\end{aligned}
$$

The first set represents the exact solution to the shell with simplysupported ends with no axial constraint (SNA-SNA). This "SNA" she11 
has boundary conditions at each end of the form

$$
\mathrm{N}_{\mathrm{x}}=0, \mathrm{v}=0, \quad \mathrm{w}=0, \mathrm{M}_{\mathrm{x}}=0 .
$$

The "CSS" set satisfles these boundary conditions term-by-term. The second set represents the exact solution to the shell with freely-supported ends with no tangential constraint (FSNT-FSNT). This "FSNT" shell has boundary conditions at each end of the form

$$
\mathbf{u}=0, \quad \hat{\mathrm{N}}_{\mathbf{x} \theta}=0, \quad \hat{\mathrm{Q}}_{\mathbf{x}}=0, \quad \frac{\partial w}{\partial \mathbf{x}}=0 .
$$

The "SCC" set satisfies the above boundary conditions term-by-term. It should be carefully pointed out that by using Stokes' transformation, it is possible to use these sets to find exact solutions for shell problems with any possible combination of homogeneous end conditions. There are sixteen possible sets of homogeneous boundary conditions that can be specified independently at each end [6]; these are 1isted in Appendix A.

\section{Stokes' Transformation}

When differentiating the foregoing series, care must be taken with respect to the end values. For example, when using a sine series to represent a function, the end values of the function are forced to be zero. With Stokes' transformation $[15,17]$, however, the end values of the sine series are released by being defined separately and these values are then included in the successive derivatives of the series.

Consider a function $f(x)$ represented by a Fourier sine series in the open range $0<x<\ell$ and by values $f_{0}$ and $f_{\ell}$ at the end points,

$$
f(0)=f_{0}, \quad f(\ell)=f_{\ell}, \quad f(x)=\sum_{k=1}^{\infty} a_{k} \sin \frac{k \pi x}{\ell}(0<x<\ell)
$$

Since it is not certain that the derivative $f^{\prime}(x)$ can be represented by term-by-term differentiation of the sine series, the derivative is instead represented by an independent cosine series of the forn 


$$
f^{\prime}(x)=b_{o}+\sum_{k=1}^{\infty} b_{k} \cos \frac{k \pi x}{\ell}
$$

Stokes' transformation then consists of integrating by parts in the basic definitions of the coefficients to obtain the required relationship between $b_{k}$ and $a_{k}$, as follows

$$
\begin{aligned}
b_{k} & =\frac{2}{\ell} \int_{0}^{\ell} f^{\prime}(x) \cos \frac{k \pi x}{\ell} d x \\
& =\frac{2}{\ell}\left[f(x) \cos \frac{k \pi x}{\ell}\right]_{0}^{\ell}+\frac{2 k \pi}{\ell^{2}} \int_{0}^{\ell} f(x)=i n \frac{k \pi x}{\ell} d x \\
& =\frac{2}{\ell}\left[(-1)^{k} f_{\ell}-f_{0}\right]+\frac{k \pi}{\ell} a_{k}
\end{aligned}
$$

Similar care must be taken when finding the correct sine series corresponding to $f^{\prime \prime}(x)$. The complete set of formulas for the sine series are then written as

$$
\begin{aligned}
& \left\{\begin{array}{l}
f(0)=f_{0} \quad f(\ell)=f_{\ell} \\
f(x)=\sum_{k=1}^{\infty} a_{k} \sin \frac{k \pi x}{\ell} \quad(0<x<\ell)
\end{array}\right. \\
& f^{\prime}(x)=-\frac{f_{0}-f_{\ell}}{\ell}-\sum_{k=1}^{\infty}\left[\frac{2}{\ell}\left\{f_{0}-(-1) f_{\ell}\right\}-\frac{\pi}{\ell} k a_{k}\right] \cos \frac{k \pi x}{\ell} \quad(0 \leq x \leq \ell) \\
& \left\{\begin{array}{l}
f^{\prime \prime}(0)=f_{0}^{\prime \prime} \quad f^{\prime \prime}(\ell)=f_{\ell}^{\prime \prime} \\
f^{\prime \prime}(x)=\left(\frac{\pi}{\ell}\right) \sum_{k=1}^{\infty} k\left[\frac{2}{\ell}\left\{f_{0}-(-1) k_{\ell}\right\}-\frac{\pi}{\ell} k a_{k}\right] \text { sin } \frac{k \pi x}{\ell}
\end{array}\right. \\
& \text { If the function } f(x) \text { is expanded in a Fourier cosine series, similar } \\
& \text { differentiated series. These formulas for the sine and cosine series are }
\end{aligned}
$$




\section{GENERAL FORMULATIONS}

The preceding theoretical considerations will now be used to solve cylindrical shell problems. The first set (6) of mode functions, CSS, identically satisfies all the boundary conditions (both geometrical and natural), only for the SNA (simple support without axial constraint) she11. Therefore, sustitution of this set into the equations of motion (1) leads to the frequency equation for an SNA shell extensively studied by Dym [18]. For all shells with other boundary conditions, the frequency equation is determined from the specified boundary conditions. Using CSS, the most complicated case (from the point of view of the size of the resulting frequency determinant) involves boundary conditions that are freelysupported without tangential constraint at both ends (FSNT-FSNT). It is interesting to note that for the second set, SCC, all the boundary conditions of the FSNT shell are satisfied on a term-by-term basis and this problem is therefore simple to solve. The most complicated problem for SCC is the SNA shell. In Appendix B, an outline is given of the set of mode functions to use to obt in the simplest frequency equation.

In the following work, details are given only for the solution using the CSS set. However, results for frequencies are shown based on both sets of axial mode functions. For maximum generality of the formulation, the FSNT shell will serve is the base problem for CSS. All cther shell problems with different boundary conditions a::e simplified special cases of this base problem. For this base problem, none of the eight boundary conditions are satisfied by cSS on a term-by-term basis. Thus the constraint conditions to satisfy these boundary conditions lead to an elghtby-eight frequency determinant. Solutions for shells with other boundary conditions lead to a smaller size determinant. 
A solution for the vibratory characteristics of an FSNT shell will now be outlined using CSS. The CSS set is given in (6) and their derivatives are obtained through the use of Stokes' transformation (see Appendix C). Substitution of the CSS set and their derivatives into the equations of motion (1) leads to an explicit relation for $A_{\text {on }}$ and, in addition, a set of equations in which $A_{k n}, B_{k n}$, and $C_{k n}$ are coupled together. The solution of these coupled equations allows all the coefficients to be written explicitly in terms of the eight unspecified quantities $\bar{u}_{0}, \bar{u}_{\ell}, v_{0}, v_{\ell}$, $w_{0}, w_{\ell}, \overline{\bar{w}}_{0}, \overline{\bar{w}}_{\ell}$ and the frequency parameter $\Omega$ as follows

$$
\begin{aligned}
& A_{\text {on }}=\frac{1}{2\left(a_{21}-\Omega\right)}\left[a_{1}\left(\bar{u}_{0}+\bar{u}_{\ell}\right)-a_{3}\left(v_{0}+v_{\ell}\right)-a_{4}\left(w_{0}+w_{\ell}\right)\right] \\
& \left(\Omega \neq \mathrm{a}_{21}\right) \\
& \left\{\begin{array}{l}
A_{k n} \\
b_{k n} \\
c_{k n}
\end{array}\right\}-\left[\begin{array}{ccc}
a_{\alpha} & a_{B} & a_{\gamma} \\
a_{\alpha} & b_{B} & b_{\gamma} \\
a_{\alpha} & b_{B} & c_{\gamma}
\end{array}\right]\left\{\begin{array}{r}
a_{1}\left[\bar{u}_{0}+\bar{u}_{\ell}(-1)^{k}\right]-a_{3}\left[v_{0}+v_{\ell}(-1)^{k}\right] \\
-a_{5} k\left[v_{0}+v_{\ell}(-1)^{k}\right] \\
-a_{7} k\left[v_{0}+v_{\ell}(-1)^{k}\right]
\end{array}\right. \\
& -a_{4}\left[w_{0}+w_{2}(-1)^{k}\right] \\
& \left.-k_{7} k i s_{0}+w_{\ell}(-1)^{k}\right] \\
& \left.-\left(a_{9} k^{3}+a_{10} k\right)\left[r_{0}+w_{\ell}(-1)^{k}\right]+a_{9} k\left[\bar{w}_{0}+\overline{\bar{w}}_{\ell}(-1)^{k}\right]\right\}
\end{aligned}
$$

$$
\left(\square_{k n} \neq 0\right)
$$

where

$$
\Omega=\left(\omega / \omega_{0}\right)^{2} \quad \omega_{0}^{2}=E / \rho R^{2}\left(1-v^{2}\right)
$$

other symbols are listed in Appendix $D$.

The equations (14) and (15) include the four unspecified quantities $v_{0}$, $v_{2}, w_{0}$, and $w_{2}$ which are directly related to the geowetrical boundary values

$$
\begin{array}{ll}
v_{0}=-\frac{2}{\pi \sin \pi \theta} v(0, \theta), & v_{2}=\frac{2}{\pi \sin n \theta} v(l, \theta) \\
w_{0}=-\frac{2}{\pi \sin n \theta} w(0, \theta) . & w_{2}=\frac{2}{\pi \sin \pi \theta} w(l, \theta)
\end{array}
$$


The other four quantities $\overline{\mathbf{u}}_{0}, \overline{\mathbf{u}}_{\ell}, \overline{\overline{\mathrm{w}}}_{0}, \overline{\overline{\mathrm{v}}}_{\ell}$ are assoclated with the unspectfied end forces $N_{x}$ and moments $M_{X X} a_{i}$ follows (see (4)),

$$
\begin{aligned}
& \left\{\begin{array}{c}
N_{x}(0, \theta) \\
-N_{x}(D, \theta)
\end{array}\right\}=-\frac{E h \ell}{2\left(1-v^{2}\right) R^{2}}\left[a_{1}\left\{\begin{array}{c}
\bar{u}_{0} \\
\bar{u}_{\ell}
\end{array}\right\}-\left(a_{3}+q_{1}\right)\left\{\begin{array}{c}
v_{0} \\
v_{\ell}
\end{array}\right\}-\left(a_{4}+q_{4}\right)\left\{\begin{array}{c}
\left.w_{0}\right) \\
w_{\ell}
\end{array}\right\}\right] \cos n \theta \\
& \left\{\begin{array}{c}
M_{x}(0, \theta) \\
-M_{x}(0, \ell)
\end{array}\right\}=\frac{E h \ell^{2}}{2 \pi\left(1-v^{2}\right) R^{2}}\left[q_{23}\left\{\begin{array}{l}
v_{0} \\
v_{\ell}
\end{array}\right\}+q_{13}\left\{\begin{array}{c}
w_{0} \\
w_{\ell}
\end{array}\right\}+a_{9}\left\{\begin{array}{c}
\bar{w}_{0} \\
\bar{w}_{\ell}
\end{array}\right\}\right] \cos n \theta
\end{aligned}
$$

The equations (14) and (15) may now be rewritten in terms of the eight unspecified boundary quantities $v_{0}, v_{\ell}, w_{0}, w_{\ell}, \mathfrak{N}_{x}^{\circ}, \mathfrak{N}_{x}^{\ell}, \mathbb{H}_{x}^{O}, \mathbb{M}_{x}^{\ell}$ for a FSNT she11.

$$
\begin{aligned}
& \left.A_{\text {on }}=\frac{1}{2\left(a_{21}-\Omega\right)}-q_{1}\left(v_{0}+v_{\ell}\right)+q_{4}\left(w_{0}+w_{\ell}\right)-\frac{2 R^{2}\left(1-v^{2}\right)}{E h \ell}\left\{N_{x}^{o}+\tilde{N}_{x}^{\ell}\right\}\right] \\
& \left(\Omega \neq \mathrm{a}_{21}\right) \\
& \left\{\begin{array}{l}
A_{k n} \\
B_{k n} \\
C_{k n}
\end{array}\right\}=\left[\begin{array}{ccc}
a_{\alpha} & a_{\beta} & a_{\gamma} \\
a_{\beta} & b_{\beta} & b_{\gamma} \\
a_{\gamma} & b_{\gamma} & c_{\gamma}
\end{array}\right]\left\{\begin{array}{l}
q_{1}\left[v_{0}+(-1)^{k} v_{\ell}\right] \\
q_{2} k\left[v_{0}+(-1)^{k} v_{\ell}\right] \\
q_{3} k\left[v_{0}+(-1)^{k} v_{\ell}\right]
\end{array}\right. \\
& +q_{4}\left[w_{0}+(-1) w_{l}^{k}\right]-\frac{2 R^{2}\left(1-v^{2}\right)}{E h l}\left[N_{x}^{2}+(-1) \hat{f}_{x}^{l \ell}\right] \\
& +q_{5}\left[w_{0}+(-1)^{k} w_{\ell}\right] \\
& +q_{6} k\left[w_{0}+(-1) w_{l}^{k}\right]-\frac{2 R^{2}\left(1-v^{2}\right)}{E h l} k\left[\tilde{M}_{x}^{20}+(-1) \frac{k M_{x}^{l}}{x}\right] \\
& \left(\square_{\mathbf{k n}} \neq 0\right)
\end{aligned}
$$

where

$$
\begin{aligned}
& \tilde{N}_{x}^{0}=\frac{N_{x}(0, \theta)}{\cos n \theta} \quad \mathcal{N}_{x}^{\ell}=-\frac{N_{x}(l, \theta)}{\cos n \theta} \\
& \tilde{H}_{x}^{0}=-\frac{\pi}{\ell \cos n \theta} M_{x}(0, \theta) \quad H_{x}^{\ell}=\frac{\pi}{\ell \cos n \theta} M_{x}(l, \theta)
\end{aligned}
$$

The boundary conditions appropriate to the FSNT shell are listed in (9). The geometric boundary conditions that must be satisfied are associated with $u$ and $\partial w / \partial x$. Hence for arbitaray $n$ it follows that 


$$
\begin{aligned}
& \psi_{u}(0)=A_{\text {on }}+\sum_{k=1}^{\infty} \cdot A_{k n}=0 \\
& \Psi_{u}(l)=A_{\text {on }}+\sum_{k=1}^{\infty} A_{k n}(-1)^{k}=0 \\
& \psi_{w}^{\prime}(0)=\frac{\pi}{\ell}\left[\frac{w_{0}+w_{\ell}}{2}+\sum_{k=1}^{\infty}\left\{w_{0}+(-1)^{k} w_{\ell}+k c_{k n}\right\}\right]=0 \\
& \left.\psi_{w}^{\prime}(l)=\frac{\pi}{\ell} \frac{\Gamma w_{0}+w_{\ell}}{2}+\sum_{k=1}^{\infty}\left\{w_{0}+(-1)^{k} w_{\ell}+k c_{k n}\right\}(-1)^{k}\right]=0 .
\end{aligned}
$$

The natural boundary conditions that must be forced to zero are $\hat{\mathrm{N}}_{\mathrm{x} \theta}=0$ and $\hat{Q}_{x}=0$ at both ends. Substitution of the CSS set and their derivatives into these equations, one obtains the following four equations

$$
\begin{aligned}
& q_{1} A_{\text {on }}+q_{2}\left(\frac{v_{o}+v_{\ell}}{2}\right)+q_{3}\left(\frac{w_{0}+w_{\ell}}{2}\right)+\sum_{k=1}^{\infty}\left[q_{1} A_{k n}+q_{2}\left\{v_{o}+v_{\ell}(-1)^{k}+k B_{k n}\right\}\right. \\
& \left.+q_{3}\left\{w_{0}+w_{\ell}(-i)^{k}+k c_{k n}\right\}\right]=0 \\
& q_{1} A_{o n}+q_{2}\left(\frac{v_{0}+v_{\ell}}{2}\right)+q_{3}\left(\frac{w_{o}+w_{\ell}}{2}\right)+\sum_{k=1}^{\infty}\left[q_{1} A_{k n}+q_{2}\left\{v_{o}+v_{\ell}(-1)^{k}+k B_{k n}\right\}\right. \\
& \left.+q_{3}\left\{w_{0}+w_{\ell}(-1)^{k}+k C_{k n}\right\}\right](-1)^{k}=0 \\
& q_{4} A_{o n}+q_{5}\left(\frac{v_{0}+v_{\ell}}{2}\right)-q_{12}\left(\frac{w_{0}+w_{\ell}}{2}\right)+a_{9}\left(\frac{\overline{\bar{w}}_{0}+\overline{\bar{w}}_{\ell}}{2}\right) \\
& +\sum_{k=1}^{\infty}\left[q_{4} A_{k n}+q_{5}\left\{v_{0}+v_{\ell}(-1)^{k}+k B_{k n}\right\}-q_{12}\left\{w_{0}+w_{\ell}(-1)^{k}+k C_{k n}\right\}\right. \\
& \left.+a_{9}\left[w_{0}+w_{\ell}(-1)^{k}-w_{0} k^{2}-w_{\ell} k^{2}(-1)^{k}-k^{3} c_{k n}\right\}\right]=0 \\
& q_{4} A_{o n}+q_{5}\left(\frac{v_{0}+v_{\ell}}{2}\right)-q_{12}\left(\frac{w_{0}+w_{\ell}}{2}\right)+a_{9}\left(\frac{w_{0}+w_{\ell}}{2}\right) \\
& +\sum_{k=1}^{\infty}\left[q_{4} A_{k n}+q_{5}\left\{v_{0}+v_{\ell}(-1)^{k}+k B_{k n}\right\}-q_{12}\left\{w_{0}+w_{\ell}(-1)^{k}+k C_{k n}\right\}\right. \\
& \left.+a_{9}\left[\bar{w}_{0}+\overline{\bar{w}}_{\ell}(-1)^{k}-w_{0} k^{2}-w_{\ell} k^{2}(-1)^{k}-k^{3} c_{k n}\right\}\right](-1)^{k}=0
\end{aligned}
$$


The last two equations contain the inexpedient $\overline{\overline{\mathrm{w}}}_{a}, \overline{\overline{\mathrm{w}}}_{\ell}$. Therefore, the equations (25) and (26) are rewritten in terms of $\tilde{M}_{X}^{\circ}$ and $\tilde{M}_{X}^{l}$ as for the case of equations (14) and (15).

$$
\begin{aligned}
& q_{4} A_{\text {on }}+q_{3}\left(\frac{v_{0}+v_{\ell}}{2}\right)-q_{18}\left(\frac{w_{0}+w_{\ell}}{2}\right)-\frac{2 R^{2}\left(1-v^{2}\right)}{E h \ell}\left(\frac{\tilde{M}_{x}^{o}+\tilde{M}_{x}}{2}\right)+\sum_{k=1}^{\infty} q_{4} A_{k n} \\
& +q_{23} k B_{k n}+q_{3}\left\{v_{o}+v_{\ell}(-1)^{k}+k B_{k n}\right\}+q_{13} k C_{k n}-a_{9}\left\{w_{o}+w_{\ell}(-1)^{k}\right. \\
& \left.+k C_{k n}\right\} k^{2}-q_{18}\left\{w_{o}+w_{\ell}(-1)^{k}+k C_{k n}\right\}-\frac{2 R^{2}\left(1-v^{2}\right)}{E h}\left\{\tilde{M}_{x}^{o}+\tilde{M}_{x}^{\ell}(-1)^{k}\right\}=0 . \\
& q_{4} A_{o n}+q_{3}\left(\frac{v_{0}+v_{\ell}}{2}\right)-q_{18}\left(\frac{w_{0}+w_{\ell}}{2}\right)-\frac{2 R^{2}\left(1-v^{2}\right)}{E h l}\left(\frac{\hat{M}_{x}^{o}+\mu_{x}^{\ell}}{2}\right)+\sum_{k=1}^{\infty} q_{4} A_{k n} \\
& +q_{23} k B_{k n}+q_{3}\left\{v_{o}+v_{\ell}(-1)^{k}+k B_{k n}\right\}+c_{13} k C_{k n}-a_{9}\left\{w_{o}+w_{\ell}(-1)^{k}\right. \\
& \left.+\mathrm{kC}_{\mathrm{kn}}\right\} \mathrm{k}^{2}-\mathrm{q}_{18}\left\{\mathrm{w}_{0}+\mathrm{w}_{\ell}(-1)^{\mathrm{k}}+\mathrm{kC}_{\mathrm{kn}}\right\}-\frac{2 \mathrm{R}^{2}\left(1-\mathrm{v}^{2}\right)}{\mathrm{Ehl}}\left\{\tilde{M}_{\mathrm{x}}^{\mathrm{o}}+\tilde{\mathrm{M}}_{\mathrm{x}}^{\ell}(-1)^{\mathrm{k}}\right\}(-1)^{\mathrm{k}}=0 \text {. }
\end{aligned}
$$

The frequency determinant may now be constructed. Substitution of the relations for the coefficients $A_{o n}, A_{k n}, B_{k n}$, and $C_{k n}(19,20)$ into the eight constraint conditions due to the geonetric and the natural boundary conditions $(22-24,27,28)$ leads to the homogeneous matr $1 x$ equation

$$
\left[e_{i, j}\right]\left\{\hat{H}_{x}^{0}, \hat{N}_{x}^{\ell}, \mathfrak{H}_{x}^{0}, \tilde{M}_{x}^{\ell}, v_{0}, v_{\ell}, w_{0}, w_{\ell}\right\}^{T}=\{0\}
$$

where $1, f=1,2, \ldots 8$.

It is interesting to compare this equation to the result previously obtained by the present author [12] through a Rayleigh-Ritz procedure in confunction with Lagrange multipliers. By redefining the unspecified end forces $\tilde{\mathbb{N}}_{\mathrm{x}}, \hat{\mathcal{H}}_{\mathrm{x}}$ as proportional to the Lagrange multipliers $\tilde{\mathbb{N}}_{\mathrm{x}}^{\mathrm{C}}=\frac{\lambda_{1}}{\pi \mathrm{R}}, \tilde{\mathbb{N}}_{\mathrm{x}}^{\ell}=\frac{\lambda_{2}}{\pi \mathrm{R}}$, $\mathrm{H}_{\mathrm{x}}^{\mathrm{O}}=\frac{\hat{\lambda}_{3}}{\mathrm{Rl}}$, and $\overrightarrow{\mathrm{H}}_{\mathrm{x}}^{\eta_{l}}=\frac{\lambda_{4}}{\mathrm{Rl}}$, equation (29) becomes identical to the matrix equation in reference [12]. For a nontrivial solution of (29), the determinant 
of the matrix must vanish,

$$
\left|e_{i, j}\right|=0
$$

resulting in a characteristic equation whose eigenvalues determine the natural frequencles of the shell. The corresponding eigenvectors determine the mode shapes. This frequency determinant is symmetric, and is based on the exact satisfaction of the boundary conditions. The natural frequency appears as a parameter $\Omega=\left(\omega / \omega_{0}\right)^{2}$ in this equation. Each element of this frequency determinant is an infinite series, and is listed in Appendix E. From the development of these equations it may be shown that there are two restrictions involved. The first restriction is $\Omega \neq \frac{1-v}{2}\left(1+\frac{\delta}{4}\right) n^{2}$. For axisymmetric $(n=0)$ shell vibrations, this becomes $\Omega \neq 0$; thereby invalidating (19) for rigid body motion. However, conditions assoriated with this rigid body motion are usually determined by inspection. For non-axisymmetric motion $(n \neq 0)$, the case of $\Omega=\frac{1-v}{2}\left(1+\frac{\delta}{4}\right) n^{2}$ is automatically rejected on the grounds that it leads to rigid body motion with non-zero frequency. The second restriction, $\square_{k n} \neq 0$, may be shown to imply that this CSS set cannot be used to solve the FSNT shell. This is not a serious drawback since it is easy to show that the second (SCC) set offers a very simple solution to this case. 


\section{FREQUENCY DETERMINANT}

In this section, the frequency determinant is derived for several cases of shell boundary conditions. The frequency determinant (30) is based on the boundary conditions of "freely-supported with no tangential constraints" at both ends (FSNT-FSNT). None of the FSNT-FSNT boundary conditions are satisfied by the CSS set of displacement functions on a term-by-term basis. The requirement to satisfy these boundary conditions by the cSS set in an overall sense led to the eight-by-eight frequency determinant (30). From this determinant, one can also obtain the frequency equation for any combination of boundary conditions among sixteen possible cases (See Appendix A) without going through the detailed procedures required to formulate the corresponding algebraic eigenvalue problems. This can be done by deleting inapplicable rows and columns from the frequency determinant (30). Therefore, solutions for shells with other boundary conditions lead to a smaller size determinant than for the FSNT-FSNT she11. For certain boundary conditions, the second set, SCC, leads to a simpler frequency determinant than the CSS set. The better choice of the mode displacement functions is suggested in Appendix B. Indeed, if the order of the frequency determinant obtained by the use of one set is "P" the one obtained by the other set is "8-P" for the same set of boundary conditions. Therefore, one can always obtain the exact frequency determinant with the order $\leq 4$ for any case of boundary conditions.

A. Simply-Supported Shell with No Axial Constraints at Both Ends (SNA-SNA)

The simplest case of boundary conditions is, of course, the SNA shell since the CSS set of axial mode functions satisfies all the boundary conditions,

$$
N_{x}=0, v=0, w=0, M_{x}=0 \quad(x=0, \ell)
$$

on a term-by-term basis. It should be noted that the boundary values 
$u, \hat{\mathbb{N}}_{x \theta}, \hat{\mathrm{Q}}_{\mathrm{x}}$, and $\frac{\partial \mathrm{w}}{\partial \mathrm{x}}$ at both ends are unspecified by this set. The frequency equation for the SNA shell is directly obtained from the equations of motion as a three-by-three determinant. The elements of the frequency determinant are no longer infinite series but reduce to algebraic terms. Past work on this problem includes that of Arnold and Warburton $[1,2]$ and Dym [18]. The frequency equation is as follows

$$
\left|\begin{array}{ccc}
s_{11} & s_{12} & s_{13} \\
& s_{22} & s_{23} \\
\text { symm. } & & s_{33}
\end{array}\right|=0 .
$$

B. Simply-Supported Shell with Axial Constraints at Both Ends (SS-SS)

As a typical case involving the enforcement of geometric boundary conditions and the release of unwanted natural boundary conditions, consider the simply-supported shell with axial constraints at both ends. The boundary conditions are

$$
u=0, v=0, w=0, \quad M_{x}=0 \quad(x=0, l) .
$$

The CSS set automatically satisfies the last three equations and the unwanted natural boundary conditions $\mathrm{N}_{\mathrm{X}}=0$ at both ends (see Eq. (31)). The frequency equation can be found from the geometrical constraint conditions of $u=0$ at both ends, and at the same instance, leaving the end forces $\mathrm{N}_{\mathrm{x}}$ unspecified. This is effectively done with (30) by retaining the rows and columns associated with $\mathrm{N}_{x}^{0}, \tilde{\mathrm{N}}_{x}^{\ell}$. The end result is the exact two-by-two frequency determinant

$$
\left|\begin{array}{cc}
e_{1,1} & e_{1,2} \\
\text { symm. } & e_{2,2}
\end{array}\right|=0 \text {. }
$$

The elements of the determinant are listed in Appendix E. It is interesting to note that the second set, SCC, would involve the enforcement and release of geometric and natural boundary conditions, and lead to a sixby-six determinant. 


\section{Shell with Clamped Ends (C-C)}

Consider the shell clamped at both ends. The boundary conditions are

$$
u=0, v=0, w=0, \frac{\partial w}{\partial x}=0 \quad(x=0, \ell) .
$$

Among these conditions, the four geometric boundary conditions $u=0$ and $\frac{\partial w}{\partial x}=0$ at both ends are not automatically satisfied by the cSS set. Instead, this CSS set unnecessarily satisfied $N_{x}=0, M_{x}=0$ at the ends. The frequency determinant is obtained from (30) by retaining the rows and columns associated with $\tilde{N}_{x}^{\circ}, \widetilde{N}_{x}^{\ell}, \widetilde{M}_{x}^{o}, \widetilde{M}_{x}^{\ell}$,

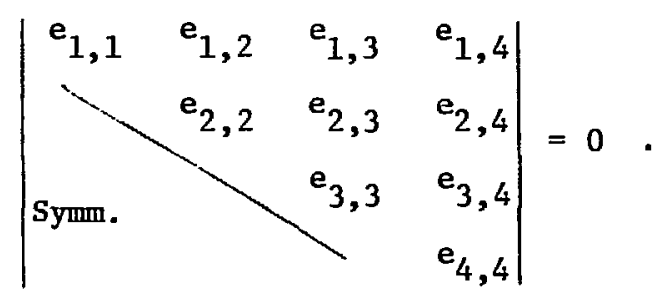

If the second set, SCC, is used, one would obtain a different four-byfour frequency determinant.

\section{Shell with Free Ends (F-F)}

As a typical case involving the enforcement of natural boundary conditions and the release of unwanted geometrical boundary conditions consider a free-free shell with boundary conditions

$$
\mathrm{N}_{\mathrm{x}}=0, \hat{\mathrm{N}}_{\mathrm{x} \theta}=0, \hat{\mathrm{Q}}_{\mathrm{x}}=0, \mathrm{M}_{\mathrm{x}}=0 \quad(\mathrm{x}=0, \ell) .
$$

From $t$ properties of CSS it is apparent that the tangential and radial displacem ts, $v$ and $w$ respectively, are identically zero at the ends. Therefore, $t$. ' releasing procedure is required to remove these unwanted geometric bouna $-\mathrm{y}$ conditions. By defining $v$ and $w$ separately at the end points (17) $a_{4}$ ' including these values in the subsequent differentiation via Stokes' transform tion, the exact frequency equation can be found from 
the enforcement of natural boundary conditions $\hat{\mathrm{N}}_{\mathrm{x} \theta}=0, \hat{\mathrm{Q}}_{\mathrm{x}}=0$ at the ends. The result is obtained from (30) by retaining the rows and columns associated with $v_{0}, v_{\ell}, w_{0}, w_{\ell}$

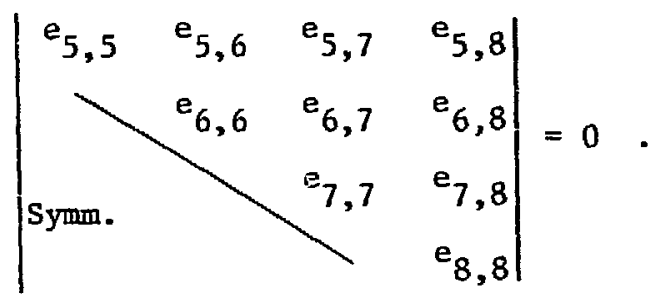

The use of the second set also leads to another four-by-four determinant.

\section{E. Clamped-Free Shel1 (C-F)}

This case involves the enforcement and release of geometrical and natural boundary conditions. For this non-symmetric set of boundary conditions

$$
\begin{aligned}
& \mathrm{u}=0, \quad \mathrm{v}=0, \quad w=0, \frac{\partial w}{\partial \mathrm{x}}=0 \quad(\mathrm{x}=0) \\
& \mathrm{N}_{\mathbf{x}}=0, \hat{\mathrm{N}}_{\mathrm{x} \theta}=0, \hat{Q}_{\mathbf{x}}=0, \mathrm{M}_{\mathbf{x}}=0 \quad(\mathrm{x}=\ell),
\end{aligned}
$$

the CSS set leads to a four-by-four frequency determinant. The $u=0$ and $\partial w / \partial x=0$ conditions at $x=0$ must be enforced with the release of the end force and moment $N_{x}, M_{x}$. The $\hat{N}_{x \theta}=0$ and $\hat{Q}_{x}=0$ conditions at $x=\ell$ must be enforced with the release of the end displacements $v, w$. Retaining the rows and columns in (30) associated with $\mathrm{N}_{\mathrm{X}}^{\circ}, \mathrm{H}_{\mathrm{X}}^{\mathrm{O}}, \mathrm{v}_{\ell}, \mathrm{v}_{\ell}$ leads to

$$
\left|\begin{array}{cccc}
e_{1,1} & e_{1,3} & e_{1,6} & e_{1,8} \\
e_{3,3} & e_{3,6} & e_{3,8} \\
e_{6,6} & e_{6,8} \\
& e_{8,8}
\end{array}\right|=0 .
$$

If the second set, SCC, were used, a different four-by-four frequency determinant would be obtained. 


\section{DISCUSSTONS OF RESULTS}

\section{A. Convergence and Accuracy}

The frequency equations derived in the former chapter involve infinite series of algebraic terms. Before any numerical results are calculated, manipulations should be performed to make the rate of convergence of the infinite series terms as close to each other as possible. The convergence of certain sums can be accelerated by subtracting off a series with a known sum [19].

As a typical example, consider the following sequence of manipulations for a simple series

$$
\begin{aligned}
\sum_{m=1}^{\infty} \frac{m^{2}}{m^{4}-\Omega} & =\sum_{m=1}^{\infty} \frac{\Omega}{\left(m^{4}-\Omega\right) m^{2}}+\sum_{m=1}^{\infty} \frac{1}{m^{2}} \\
& =\sum_{m=1}^{\infty} \frac{\Omega^{2}}{\left(m^{4}-\Omega\right) m^{6}}+\Omega \sum_{m=1}^{\infty} \frac{1}{m^{6}}+\sum_{m=1}^{\infty} \frac{1}{m^{2}} \\
& =\sum_{m=1}^{\infty} \frac{\Omega^{2}}{\left(m^{4}-\Omega\right) m^{6}}+\frac{\pi^{6}}{945} \Omega+\frac{\pi^{2}}{6} .
\end{aligned}
$$

Through the above process, the order of the series has been improved from $\mathrm{m}^{-2}$ to $\mathrm{m}^{-10}$, and accordingly, the rate of convergence will be greatly accelerated.

The infinite series in the shell frequency determinant were treated in the same way as in the above example. In this way, fast convergence was obtained and the same upper limit could be used for all series in the frequency determinant; typically an upper limit of 50 terms was used. Frequency results were obtained from the frequency determinant by substituting values of $\omega / \omega_{0}$ and monitoring the determinant until it vanished.

Convergence and accuracy of the present method is described in Tables

1 and 2. In order to facilitate comparison with the exact solutions reported by other contributors who used Flügge's and Donne11's theory, 
Table 1. Convergence of Natural Frequency Parameters, $w_{0} \omega_{0}$

\begin{tabular}{|c|c|c|c|c|c|c|c|c|}
\hline \multirow{2}{*}{$\begin{array}{l}\text { Number } \\
\text { of } \\
\text { Terms }\end{array}$} & \multicolumn{2}{|c|}{ Case 1} & \multicolumn{2}{|c|}{ Case 2} & \multicolumn{2}{|c|}{ Casse 3} & \multicolumn{2}{|c|}{ Case 4} \\
\hline & Sanders & Donnell & Sanders & Donnell & Sanders & Donneil & Sanders & Donnell \\
\hline 5 & .01523 & .01555 & .05880 & .0684 & .3161 & .3230 & .3589 & .3594 \\
\hline 7 & .01520 & .01552 & .05851 & .0681 & .3134 & .3203 & .3586 & .3591 \\
\hline 10 & .01517 & .01550 & .05823 & .06792 & .3122 & .3191 & .3585 & .3590 \\
\hline 20 & .01515 & .01547 & .05795 & .05767 & .3119 & .3188 & .3585 & .3590 \\
\hline 30 & .01513 & .01545 & .05788 & .06760 & .3119 & .3188 & .3585 & .3590 \\
\hline 40 & .01512 & .01544 & .05785 & .06758 & .3119 & .3188 & .3585 & .3590 \\
\hline 50 & .01510 & .01542 & .05784 & .06758 & .3118 & .3188 & .3585 & .3590 \\
\hline 100 & .01509 & .01541 & .05784 & .06757 & .3118 & .3188 & .3585 & .3590 \\
\hline 150 & .01509 & .01541 & $.0578 \dot{4}$ & .06757 & .3118 & .3188 & .3585 & .3590 \\
\hline 200 & .01509 & .01541 & .05784 & .06757 & .3118 & .3188 & .3585 & .3590 \\
\hline 300 & .01508 & .01541 & .05784 & $.0675 ?$ & .3118 & $.3 .9 \%$ & .3585 & .3590 \\
\hline 500 & .01508 & .01541 & .05784 & .06757 & . 3118 & .3188 & .3585 & .3590 \\
\hline Ref. [19] & $.01508^{\star}$ & .01541 & $.05787^{\star}$ & .06757 & $.3117^{k}$ & .3188 & $.3583^{\star}$ & $\hbar \star$ \\
\hline
\end{tabular}

Case 1: Clamped-Clamped Shell $(\ell / R=10, R / h=500, v=0.3, n=4)$

Case 2: Clamped-Clamped Shell $(l / R=10, R / h=20, v=0.3, \mathfrak{n}=2)$

Case 3: Clamped-C.Lamped shell $(\ell / R=2, R / h=20, v=0.3, n=3)$

Case 4: Free-Free Shell $\quad(\ell / R=5, R / h=20, v=0.3, n=1)$

* Solutions based on Flügge's theory $[7,8]$.

${ }^{\star *}$ No exact solutions are available from literature. 
Table 2. Comparison of Natural Frequency Parameters, w/

\begin{tabular}{|c|c|c|c|c|c|c|c|c|c|}
\hline \multirow{2}{*}{$n$} & \multirow{2}{*}{$m$} & \multirow{2}{*}{$\begin{array}{l}\text { Boundary } \\
\text { Condition }\end{array}$} & \multicolumn{3}{|c|}{ Shell Paramecers } & \multicolumn{2}{|c|}{ Prescnt Method } & \multicolumn{2}{|c|}{ orhers ${ }^{*}$} \\
\hline & & & $2 / R$ & $\mathbf{R} / \mathbf{h}$ & ע & Sanders & Donnell & Pltigge & Donnell \\
\hline 4 & 1 & $c-c$ & 10 & 500 & 0.3 & .01508 & .01541 & .01508 & .01541 \\
\hline 2 & 1 & $\mathrm{C}-\mathrm{C}$ & 10 & 20 & 0.3 & .05784 & .06757 & .05787 & .06757 \\
\hline 3 & 1 & $\mathrm{C}-\mathrm{C}$ & 2 & 20 & 0.3 & .3118 & .3188 & .3117 & .3188 \\
\hline 1 & 1 & $F-F$ & 5 & 20 & 0.3 & .3585 & .3590 & .3583 & \\
\hline 0 & 1 & $F-F$ & 8.1 & 20 & 0.3 & .3669 & & .3671 & \\
\hline 2 & 5 & $F-F$ & 8.67 & 500 & 0.3 & .4472 & & .4472 & \\
\hline 2 & 1 & $C-F$ & 1.14 & 20 & 0.3 & .3076 & & .3082 & \\
\hline 2 & 2 & $c-F$ & 2.88 & 20 & 0.3 & .3081 & & .3082 & \\
\hline 2 & 3 & $C-F$ & 5.07 & 20 & 0.3 & .3079 & & .3082 & \\
\hline 4 & 1 & Ss-ss & 10 & 500 & 0.3 & .01508 & & .01507 & \\
\hline 1 & 1 & SNAT-SNAT & 30 & 500 & 0.3 & .00702 & & .00700 & \\
\hline 1 & 3 & SNAT-SNAT & 5 & 500 & 0.3 & .3699 & & .3698 & \\
\hline 0 & 1 & SNA-SRA & $2 \pi$ & 10 & 0.3 & .296081 & .295804 & $.296081^{\hbar k}$ & .295804 \\
\hline 2 & 1 & SWA-SNA & $2 \pi$ & 10 & 0.3 & .095812 & .119730 & $.095812^{\star \star}$ & .119730 \\
\hline 4 & 1 & SNA-SNA & $2 \pi$ & 10 & 0.3 & .425921 & .454809 & $.425921^{k \star}$ & .454808 \\
\hline
\end{tabular}

*These results are due to Forsberg $[6,7,8]$, Warburton $[9,10\}$ and Dym [18].

"these solutions are based on Sanders' theory $\{18]$. 
the present method was also applied to Jonnell's equations. In general, the solution approaches the exact frequency from above as th? nimber of terms included in the series increases. However, as shown in Table 1, the use of more than 50 terms did not improve the solutions significantly. Table 2 compares the results of the present method with the available exact solutions. In all cases there was excellent comparison for a wide variety of boundary conditions and geometric parameters. For shells clamped at both ends, the solutions of the present method using Donnel1's theory are the same as those reported by Forsberg [8]. The frequency parameters for the SNA-SNA shell by the use of Sanders' as well as Donnell's theory are also identical to the results given by Dym [18]. For other boundary conditions, F-F, C-F, SS-SS and SNAT-SNAT, the available soluthons are all based on Flügge's shell theory. The differences between solutions obtained from different thin shell theories are usually negligible as revealed in Table 2 .

Node shapes for a particular natural frequency can always be investigated by substituting the calculated frequency parameter into the frequency matrix and finding the relative values of unspecified end forces and values, and finally evalualing the coefficients of the assumed modal displacement functions. Comparison of mode shapes between the present method and others is not attempted in this report.

\section{B. Vibrations of Thermal Liner}

The thermal liner of the Fast Test Reactor (FTR) is a thin, circular cylindrical shell situated concentrically inside the main reactor vesse1. The annular gap between these two cylindrical vessels is occupied by sodium coolant. Since the thermal liner is subjected to sodium coolant flow, it is susceptible to flow-induced vibrations. A vibration problem 
may occur if the natural frequencies of the thermal liner are close to the frequency of some vibration source in the reactor vessel. The support conditions of the thermal liner may be best represented as rigidly fixed at the bottom and free at the top: clamped-free (C-F) boundary conditions.

A series of experiments had been performed as a part of an effort to investigate the modal characteristics of the thermal liner [20]. The experimental model is approximately $1 / 14$ scale of the prototype thermal liner for the FTR, giving the following dimensions and material properties:

Thickness $h=0.058$ in.

Radius (to the center

$$
\text { of thickness) } \quad R=8.505 \text { in }
$$

Length

$\ell=20.125 \mathrm{in}$.

Materia1 density

$\rho=701 \times 10^{-6} 1 \mathrm{~b}-\sec ^{2} /$ in. ${ }^{4}$

Young's modulus

$E=26.5 \times 10^{6} \mathrm{psi}$

Poisson's ratio

$v=0.3$

The experimental model was intended to simulate a clamped-free she11; however, insufficient clamping $(\partial w / \partial x \neq 0)$ and imperfect axial constraint $(u \neq 0)$ at the bottom has been judged a probable cause for disagreement between the experimental results and the analytical predictions of natural frequencies. Therefore, in this study the sensitivity of the natural frequencies to changes in the bottom-boundary conditions is investigated using the exact present solution method. The accuracy of the solutions is compared to the results obtained by two finite element computer codes: SAP IV [21] and NASTRAN [22]. The boundary conditions considered are:
(1) C-F : Clamped-Free
(2) SS-F : Simply Supported-Free 
(3) CNA-F: Clamped with No Axtal Constrain-Free

(4) SNA-F: Simply Supported with No Axial Conatrain-Free.

In Table 3 the solutions of the present gethod are compared to the SAP IV and NASTRAN results for the theral lingr with ciamped-free boundary conditions. The finite element grid used with the two computer codes is the same and conslsts of 10 divisions vercically and 9 divisions over a quarter of the shell circumferentially. Apparently the present exact method always gives lower values caupared to the two finite element computer codes. The solutions of SAP IV are beter than those of NASTRAN, as shown by their closeness to the results of the present methud, because SAP IV uses a more accurate quadrilateral element.

The natural frequencies for the thermal liner model in vacuum are calculated by the present method for the ahove four cases of boundary conditions, and sumarized in Table 4 and Fig. 2. The frequencies become smaller as the boundary conditions change from Case (1) to Case (4) for a given circunferential and an ixial mode number, $n$ and $m$. The ef fect of clamping $(\partial w / \partial x=0)$ can be evaluated by comparing the frequencies for the two pairs of boundary conditions: $C-F$ and SS-F, CNA-F and SNA-F. The natural frequencies for $S S-F$ are smaller than those for C-F by about less than $1 \%$; thus they are not distinguishable in Fig. 2. The differences between frequencies for $\mathrm{CNA}-\mathrm{F}$ and SNA-F are also small $(<1.5 \%)$, except for the modes of $n=0$ to 3 with $m=1$. The influence of axial constraint $(u=0)$ is evident in Fig. 2 and, in contrast to the effect of clamping, is significant throughout most of the region of interest. Note that the frequency curve for $m=1$ is great $1 y$ shifted down for $n<5$; moreover, the lowest natural frequencies for shell-type vibration ( $\geq 2$ ) occur at $\mathrm{n}=2$ for CNA-F and SNA-F, in contrast to $\mathrm{n}=4$ for $C-F$ and $S S-F$. However, for higher axial half-waves $(m=2,3)$, the differences are less than $20 \%$ 
Table 3. Comparison of Natural Frequencies (Hz) Eor the Thermal Liner with Clamped=Free Boundary Conditions

\begin{tabular}{|c|c|c|c|c|}
\hline $\mathbf{n}$ & a & Present Method & SAP IV & NASTRAN \\
\hline 1 & 1 & 855.10 & & 856.3 \\
\hline 2 & 1 & 403.72 & 405.1 & 410.1 \\
\hline 3 & 1 & 223.34 & 225.6 & 232.2 \\
\hline 4 & 1 & 171.77 & 174.3 & 180.5 \\
\hline 5 & 1 & 199.16 & 201.7 & 206.2 \\
\hline 6 & 1 & 268.86 & 272.0 & 275.5 \\
\hline 7 & 1 & 361.92 & 366.2 & 370.1 \\
\hline 8 & 1 & 472.54 & 478.4 & 483.5 \\
\hline 9 & 1 & 599.03 & & 614.0 \\
\hline 3 & 2 & 928.28 & & 943.2 \\
\hline 4 & 2 & 644.48 & & 671.4 \\
\hline 5 & 2 & 494.69 & 505.8 & 529.3 \\
\hline 6 & 2 & 442.00 & 454.3 & 478.0 \\
\hline 7 & 2 & 464.59 & 476.7 & 496.9 \\
\hline 8 & 2 & 539.45 & & 567.2 \\
\hline 9 & 2 & 648.34 & & 673.2 \\
\hline
\end{tabular}


Table 4. Natural Frequencies $(\mathrm{Hz})$ of Thermal Liner for Various Boundary Conditions

\begin{tabular}{|c|c|c|c|c|c|}
\hline$n$ & $\underline{m}$ & $\mathrm{C}-\mathrm{E}$ & SS-F & CNA-F & SNA-F \\
\hline 0 & 1 & 1497.90 & 1497.90 & 0 & 0 \\
\hline 1 & 1 & 855.10 & 853.85 & 12.50 & 0 \\
\hline 2 & 1 & 403.72 & 402.59 & 27.79 & 21.57 \\
\hline 3 & 1 & 223.34 & 222.61 & 61.81 & 58.93 \\
\hline 4 & 1 & 171.77 & 171.34 & 112.92 & 111.32 \\
\hline 5 & 1 & 199.16 & 198.63 & 179.68 & 178.68 \\
\hline 6 & 1 & 268.86 & 268.72 & 261.69 & 261.03 \\
\hline 7 & 1 & 361.92 & 361.82 & 358.81 & 358.34 \\
\hline 8 & 1 & 472.54 & 472.45 & 470.97 & 470.63 \\
\hline 9 & 1 & 599.03 & 598.94 & 598.15 & 597.89 \\
\hline 10 & 1 & 740.81 & $740.7 ?$ & 740.32 & 740.12 \\
\hline 0 & 2 & 2351.51 & 2346.69 & 1497.90 & 1497.90 \\
\hline 1 & 2 & 2318.98 & 2318.98 & 2077.48 & 2077.24 \\
\hline 2 & 2 & 1437.11 & 1435.35 & 1408.98 & 1407.79 \\
\hline 3 & 2 & 928.28 & 925.39 & 844.59 & 842.44 \\
\hline 4 & 2 & 644.48 & 641.43 & 552.22 & 548.87 \\
\hline 5 & 2 & 494.69 & 491.99 & 418.54 & 414.14 \\
\hline 6 & 2 & 442.00 & 439.89 & 390.44 & 385.77 \\
\hline 7 & 2 & 464.59 & 463.06 & 434.21 & 430.04 \\
\hline 8 & 2 & 539.45 & 538.31 & 522.17 & 518.70 \\
\hline 9 & 2 & 648.34 & 547.41 & 638.16 & 635.28 \\
\hline 10 & 2 & 781.15 & 780.37 & 774.87 & 772.44 \\
\hline 1 & 3 & 3076.05 & 3070.64 & 2723.53 & 2723.92 \\
\hline 2 & 3 & 2487.60 & 2484.64 & 2479.20 & 2476.84 \\
\hline 3 & 3 & 1834.82 & 1830.35 & 1811.52 & 1808.06 \\
\hline 4 & 3 & 1367.64 & 1361.83 & 1323.62 & 1318.80 \\
\hline 5 & 3 & 1057.12 & 1050.67 & 1001.38 & 995.01 \\
\hline 6 & 3 & 864,82 & 858.43 & 308.93 & 801.14 \\
\hline 7 & 3 & 767.65 & 761.85 & 720.27 & 711.69 \\
\hline 8 & 3 & 750.67 & 745.75 & 715.49 & 707.05 \\
\hline 9 & 3 & 798.18 & 794.11 & 774.21 & 766.53 \\
\hline 10 & 3 & 893.60 & 890.18 & 877.72 & 871.04 \\
\hline
\end{tabular}




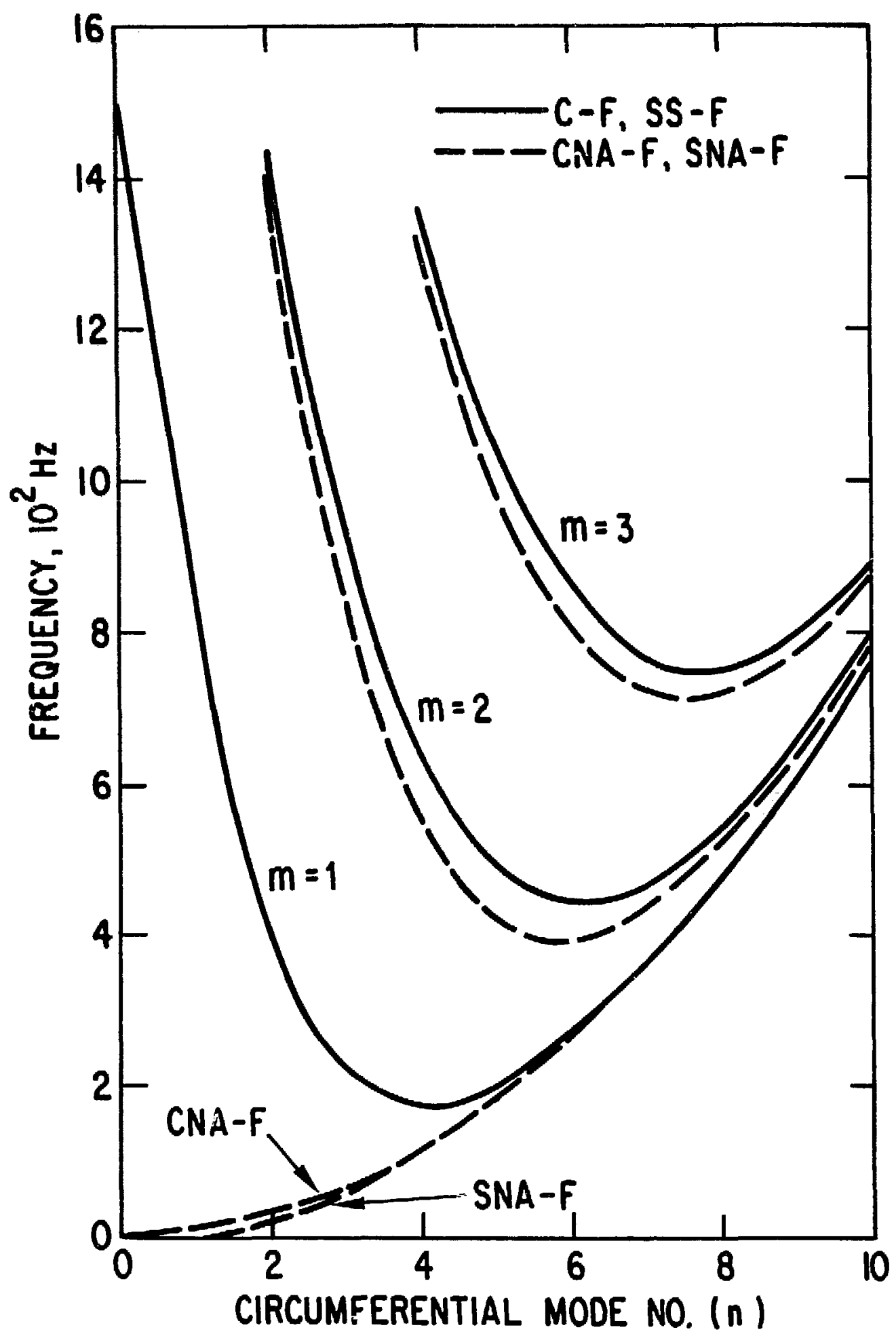

Fig. 2. Natural Frequencies of Thermal Liner Model 
throughout the entire range of circumferential wave numbers $n$. A comparative study of the natural frequencies between the analytfcal predictions and experimental results is not considered to be within the scope of the present report and is reported elsewhere [20]. 
vI. CONCLUSIONS

In this report, a general solution procedure for the free vibration of a circular cylindrical shell is presented. The present method offers distinct computational advantages over other exact solution methods [6-11]. Table 5 compares the present method and other exact solution procedures. An uncoupled explicit expression of frequency determinant is obtained by directly solving Sanders' shell equations. The frequency equation is obtained in the process of satisfying all boundary conditions exactly by the assumed simple Fourier series. This frequency equation involves infinite series which are evaluated by the use of appropriate numerical techniques and a computer. The order of frequency determinant may vary from 1 to 8 depending on the boundary conditions to be satisfied. However, an appropriate choice of displacement functions (i.e., the CSS or SCC set) can always furnish a frequency determinant of low order ( $\leq 4)$ for any given boundary conditions. The frequency determinant obtained by others [6-11] is always eight-by-eight and coupled with eighth-order algebraic equations resulting from the shell equations. The elements of frequency determinant consist of transcendental functions in place of the infinite series of the present method. However, it should be noted that the transcendental function is evaluated as a series in the present day computer.

In conclusion, the method presented in this report is a relatively simple, exact-solution method for the free vibration problem of circular cylindrical shells. 
Table 5. Comparisons of the Present Method with the other Exact Solution Method

\begin{tabular}{|c|c|c|c|}
\hline & Items & Present Method & Others * \\
\hline 1. & $\begin{array}{l}\text { Frequency } \\
\text { Determinant }\end{array}$ & $\begin{array}{l}\text { Uncoupled } \\
\text { explicit expression }\end{array}$ & $\begin{array}{l}\text { Coupled with } \\
\text { 8th order } \\
\text { algebraic equation }\end{array}$ \\
\hline 2. & $\begin{array}{l}\text { Order of Frequency } \\
\text { Determinant }\end{array}$ & $\begin{array}{l}\text { Varies }(1 \backsim 4) \\
\text { dependent on } \\
\text { boundary conditions }\end{array}$ & Fixed (8) \\
\hline 3. & $\begin{array}{l}\text { Element of } \\
\text { Frequency Determinant }\end{array}$ & $\begin{array}{l}\text { Infinite series } \\
\text { (rate of conver- } \\
\text { gence can be } \\
\text { accelerated) }\end{array}$ & $\begin{array}{l}\text { Transcendental } \\
\text { Functions } \\
\text { (also series in computer } \\
\text { computation) }\end{array}$ \\
\hline
\end{tabular}

${ }_{\text {Ref erences [6-11] }}$ 
APPENDIX A

Boundary Conditions of Circular Cylindrical Shell

\begin{tabular}{|c|c|c|c|c|c|}
\hline Case & Code & Description & \multicolumn{3}{|c|}{$\begin{array}{l}\text { Boundary } \\
\text { Conditions }\end{array}$} \\
\hline 1 & $\mathrm{C}$ & Clamped & $\mathbf{w}$ & $\partial w / \partial x$ & $\mathbf{u} \quad \mathbf{v}$ \\
\hline 2 & CNA & $\begin{array}{l}\text { Clamped with no Axial } \\
\text { Constraints }\end{array}$ & $w$ & $\partial w / \partial x$ & $\mathbf{N}_{\mathbf{x}} \mathrm{v}$ \\
\hline 3 & CNT & $\begin{array}{l}\text { Clamped with no Tangential } \\
\text { Constraints }\end{array}$ & $\mathbf{W}$ & $\partial w / \partial x$ & $\mathrm{u} \quad \hat{\mathrm{N}}_{\mathrm{x} \theta}$ \\
\hline 4 & CNAT & $\begin{array}{l}\text { Clamped with no Axial, } \\
\text { Tangential Constraints } \\
\end{array}$ & $\mathbf{w}$ & $\partial w / \partial x$ & $\mathrm{~N}_{\mathrm{x}} \hat{\mathrm{N}}_{\mathrm{x} \theta}$ \\
\hline 5 & ss & Simply-Supported & $\mathbf{w}$ & $\mathrm{M}_{\mathrm{X}}$ & $\mathbf{u} \mathbf{v}$ \\
\hline 6 & SNA & $\begin{array}{l}\text { Simply-Supported with no Axial } \\
\text { Constraints (Shear Diaphragm) }\end{array}$ & $\mathbf{w}$ & $\mathbf{M}_{\mathbf{x}}$ & $\mathbb{N}_{x} \mathbf{v}$ \\
\hline 7 & SNT & $\begin{array}{l}\text { Simply-Supported with no } \\
\text { Tangential Constraints }\end{array}$ & $\mathbf{w}$ & $\mathbf{M}_{\mathrm{x}}$ & $\mathbf{u} \hat{\mathrm{N}}_{\mathbf{x} \theta}$ \\
\hline 8 & SNAT & $\begin{array}{l}\text { Simply-Supported with no } \\
\text { Axial, Tangential Constraints }\end{array}$ & $\mathbf{w}$ & $\mathrm{M}_{\mathbf{x}}$ & $\mathrm{N}_{\mathbf{x}} \hat{\mathrm{N}}_{\mathrm{x} \theta}$ \\
\hline 9 & FS & Freely-Supported & $\hat{\mathrm{q}}_{\mathrm{x}}$ & $\partial w / \partial \mathbf{x}$ & $\mathbf{u} \mathbf{v}$ \\
\hline 10 & FSNA & $\begin{array}{l}\text { Freely-Supported with no } \\
\text { Axia1 Constraints }\end{array}$ & $\hat{\mathrm{Q}} \mathrm{x}$ & $\partial w / \partial x$ & $N_{x} v$ \\
\hline 11 & FSNP & $\begin{array}{l}\text { Freely-Supported with no } \\
\text { Tangential Constraints }\end{array}$ & $\hat{\mathrm{Q}}$ & $\partial w / \partial x$ & $\mathbf{u} \hat{\mathrm{N}}_{\mathbf{x} \theta}$ \\
\hline 12 & FSNAT & $\begin{array}{l}\text { Freely-Supported with no } \\
\text { Axial, Tangential Constraints }\end{array}$ & $\hat{\mathrm{q}}_{\mathrm{x}}$ & $\partial w / \partial x$ & $\mathrm{~N}_{\mathrm{x}} \hat{\mathrm{N}}_{\mathrm{x} \theta}$ \\
\hline 13 & FAT & $\begin{array}{l}\text { Free with Axial, Tangential } \\
\text { Constraints }\end{array}$ & $\hat{Q}_{x}$ & $\mathrm{M}_{\mathrm{x}}$ & $\mathbf{u} \mathbf{v}$ \\
\hline 14 & FT & $\begin{array}{l}\text { Free with Tangential } \\
\text { Constraints }\end{array}$ & $\hat{\mathrm{Q}}$ & $\mathrm{M}_{\mathbf{x}}$ & $\mathrm{N}_{\mathrm{x}} \mathrm{v}$ \\
\hline 15 & FA & Free with Axial Constraints & $\hat{\mathrm{Q}}_{\mathrm{x}}$ & $\mathrm{M}_{\mathrm{x}}$ & $\mathrm{u} \hat{\mathrm{N}}_{\mathrm{x} \theta}$ \\
\hline 16 & $\mathbf{F}$ & Free & $\hat{Q}_{\mathbf{x}}$ & $M_{x}$ & $\mathrm{~N}_{\mathrm{x}} \hat{\mathrm{N}}_{\mathrm{xe}}$ \\
\hline
\end{tabular}


APPENDIX B

Shell Boundary Conditions to be Satisfied

on using the First (CSS) or the Second (SCC) Set

\begin{tabular}{|c|c|c|c|c|c|c|c|}
\hline \multirow{2}{*}{$\frac{\text { Case }}{1}$} & \multirow{2}{*}{$\frac{\text { Code }}{C}$} & \multicolumn{2}{|c|}{ First Set (css) } & \multicolumn{3}{|c|}{ Second Set (SCC) } & \multirow{2}{*}{$\begin{array}{c}\begin{array}{c}\text { Better } \\
\text { Choice }\end{array} \\
1,2\end{array}$} \\
\hline & & $\mathbf{u}$ & $\partial w / \partial x$ & $\mathbf{v}$ & $\mathbf{w}$ & & \\
\hline 2 & CNA & & $\partial w / \partial x$ & $\mathbf{v}$ & $\boldsymbol{w}$ & $\mathrm{N}_{\mathrm{x}}$ & 1 \\
\hline 3 & CNT & $\mathbf{u}$ & $\partial w / \partial x \quad \hat{\mathbb{N}}_{x \theta}$ & & $w$ & & 2 \\
\hline 4 & CNAT & & $\partial w / \partial \mathbf{x} \quad \hat{\mathbf{N}}_{\mathbf{x} \theta}$ & & 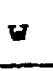 & $\mathbf{N}_{\mathbf{x}}$ & 1,2 \\
\hline 5 & SS & $\mathbf{u}$ & & $\mathbf{v}$ & $\mathbf{w}$ & $M_{x}$ & 1 \\
\hline 6 & SNA & & - & $\mathbf{v}$ & $\mathbf{w}$ & $N_{x} \quad M_{x}$ & 1 \\
\hline 7 & SNT & $\mathbf{u}$ & $\hat{\mathrm{N}}_{\mathrm{x} \theta}$ & & $w$ & $M_{x}$ & 1,2 \\
\hline 8 & SNAT & & $\hat{\mathrm{N}}_{\mathbf{x} \theta}$ & & w & $N_{x} \quad M_{x}$ & 1 \\
\hline 9 & FS & $\mathbf{u}$ & $\partial w / \partial x$ & $\mathbf{v}$ & & & 2 \\
\hline 10 & ESNA & & $\partial w / \partial \mathbf{x}$ & $\mathbf{v}$ & & $\mathrm{N}_{\mathbf{x}}$ & 2 \\
\hline 11 & FSNT & $\mathbf{u}$ & $\partial w / \partial \mathbf{x} \quad \hat{\mathbf{N}}_{\mathbf{x} \theta}$ & & . & & 2 \\
\hline 12 & FSNAT & & $\partial w / \partial \mathbf{x} \quad \hat{\mathbb{N}}_{\mathbf{x} \theta}$ & & & $\mathrm{N}_{\mathbf{x}}$ & 2 \\
\hline 13 & FAT & $\mathbf{u}$ & & $\mathbf{v}$ & & $\mathrm{M}_{\mathrm{X}}$ & 2 \\
\hline 14 & ET & & & $\mathbf{v}$ & & $\mathrm{N}_{\mathrm{X}} \quad \mathrm{M}_{\mathrm{X}}$ & 1 \\
\hline 15 & FA & $\mathbf{u}$ & $\hat{\mathbf{N}}_{\mathbf{x} \theta}$ & & & $\mathrm{M}_{\mathrm{x}}$ & 2 \\
\hline 16 & F & & $\hat{\mathrm{N}}_{\mathbf{x} \in}$ & & & $N_{x} \quad M_{x}$ & 2 \\
\hline
\end{tabular}




\section{APPENDIX C}

\section{First Set (CSS) of Displacement Functions and Their Derivatives}

$$
\begin{aligned}
& u(x, \theta)=\left(A_{\text {on }}+\sum_{k=1}^{\infty} A_{k n} \cos \frac{k \pi x}{\ell}\right) \cos n \theta \quad(0 \leq x \leq \ell) \\
& u_{, x}=-\left(\frac{\pi}{\ell}\right) \sum_{k=1}^{\infty} A_{k n} k \sin \frac{k \pi x}{\ell} \cos n \theta \quad(0<x<\ell) \\
& u_{, x}(0, \theta)=-\left(\frac{\pi^{2}}{2 \ell}\right) \bar{u}_{0} \cos n \theta \\
& u_{, x}(\ell, \theta)=\left(\frac{\pi^{2}}{2 \ell}\right) \bar{u}_{\ell} \cos n \theta \\
& u_{, x x}=\left(\frac{\pi}{\ell}\right)^{2}\left[\frac{\bar{u}_{0}+u_{\ell}}{2}+\sum_{k=1}^{\infty}\left[\bar{u}_{0}+\bar{u}_{\ell}(-1)^{k}-k^{2} A_{k n}\right\} \cos \frac{k \pi x}{\ell}\right] \cos n \theta \quad(0 \leq x \leq \ell) \\
& v(x, \theta)=\sum_{k=1}^{\infty} B_{k n} \sin \frac{k \pi x}{\ell} \sin n \theta \quad(0<x<\ell) \\
& v(0, \theta)=-\frac{\pi}{2} v_{0} \sin n \theta \\
& v(\ell, \theta)=\frac{\pi}{2} v_{\ell} \sin n \theta \\
& v_{, x}=\left(\frac{\pi}{\ell}\right)\left[\frac{v_{0}+v_{\ell}}{2}+\sum_{k=1}^{\infty}\left\{v_{0}+v_{\ell}(-1)^{k}+k B_{k n}\right\} \cos \frac{k \pi x}{\ell}\right] \sin n \theta \quad(0 \leq x \leq \ell) \\
& v_{, x x}=-\left(\frac{\pi}{\ell}\right)^{2}\left[\sum_{k=1}^{\infty}\left\{v_{0} k+v_{\ell} k(-1)^{k}+k^{2} B_{k n}\right\} \sin \frac{k \pi x}{\ell}\right] \sin n \theta \quad(0<x<\ell) \\
& v_{, x x}(0, \theta)=-\frac{\pi^{3}}{2 \ell^{2}} \overline{\bar{v}}_{0} \sin n \theta \\
& v, x x(\ell, \theta)=\frac{\pi^{3}}{2 \ell^{2}} \overline{\bar{v}}_{\ell} \sin n \theta
\end{aligned}
$$


42

$$
\begin{aligned}
& w(x, \theta)=\sum_{k=1}^{\infty} C_{k n} \sin \frac{k \pi x}{\ell} \cos n \theta \quad(0<x<\ell) \\
& w(0, \theta)=-\frac{\pi}{2} \omega_{0} \cos n \theta \\
& w(l, \theta)=\frac{\pi}{2} w_{2} \cos n \theta \\
& w_{, x}=\left(\frac{\pi}{\ell}\right)\left[\frac{w_{0}+w_{\ell}}{2}+\sum_{k=1}^{\infty}\left\{w_{0}+w_{\ell}(-1)^{k}+k \cdot c_{k n}\right\} \cos \frac{k \pi x}{\ell}\right] \cos n \theta \quad(0 \leq x \leq \ell) \\
& w_{, x x}=-\left(\frac{\pi}{\ell}\right)^{2}\left[\sum_{k=1}^{\infty}\left\{w_{0} k+w_{\ell} k(-1)^{k}+k^{2} c_{k n}\right\} \sin \frac{k \pi x}{\ell}\right] \cos n \theta \quad(0<x<\ell) \\
& w, x x^{(0, \theta)}=-\frac{\pi^{3}}{20^{2}} \overline{\bar{w}}_{0} \cos n \theta \\
& w, x x(\ell, \theta)=\frac{\pi^{3}}{2 \ell^{2}} \overline{\bar{\omega}}_{\ell} \cos n \theta \\
& { }_{, x 00 x}=\left(\frac{\pi}{\ell}\right)^{3}\left[\frac{\overline{\bar{w}}_{0}+\overline{\bar{w}}_{\ell}}{2}+\sum_{k=1}^{\infty}\left\{\overline{\bar{w}}_{0}+\overline{\overline{\mathrm{w}}}_{\ell}(-1)^{k}-w_{0} k^{2}-w_{\ell} k^{2}(-1)^{k}\right.\right. \\
& \left.\left.-k^{3} c_{k n}\right\} \cos \frac{k \pi x}{\ell}\right] \cos n \theta \quad(0 \leq x \leq \ell) \\
& { }_{, \operatorname{xxxx}}=\left(\frac{\pi}{\ell}\right)^{4}\left[\sum _ { k = 1 } ^ { \infty } \left\{-\overline{\bar{w}}_{0} k-\overline{\bar{w}}_{\ell} k(-1)^{k}+w_{0} k^{3}+w_{\ell} k^{3}(-1)^{k}\right.\right. \\
& \left.\left.+k^{4} C_{k n}\right] \sin \frac{k \pi x}{\ell}\right] \cos n \theta \quad(0<x<\ell) \\
& { }^{w}, \operatorname{xx\times x}(0, \theta)=\overline{\overline{\bar{w}}}_{o} \cos n \theta \\
& { }^{w}, \operatorname{xxxx}(\ell, \theta)=\overline{\bar{F}}_{\ell} \cos n \theta
\end{aligned}
$$


43

APPENDIX D

Symbols used in Equations (14)-(28) and Appendix E

$$
\begin{aligned}
& a_{1}=(\mathrm{rR} / \ell)^{2}, \quad a_{21}=\frac{1-\nu}{2}\left(1+\frac{\delta}{4}\right) n^{2}, \quad a_{2}=a_{21}-\Omega \\
& a_{3}=-\frac{\pi R}{\ell}\left[\frac{1+\nu}{2}-\frac{3(1-\nu)}{8} \delta ! n, \quad a_{4}=-\frac{\pi R}{\ell}\left[v-\frac{(1-v)}{2} \delta n^{2}\right]\right. \\
& a_{5}=\left(\frac{\pi R}{l}\right)^{2} \frac{1-v}{2}\left(1+\frac{9}{4} \delta\right), \quad a_{61}=(1+\delta) n^{2}, \quad a_{6}=a_{E 1}-\Omega \\
& a_{7}=\left(\frac{\pi R}{l}\right)^{2} \frac{3-v}{2} \delta n, \quad a_{8}=\left(1+\delta n^{2}\right) n, \quad a_{9}=\left(\frac{\pi R}{l}\right)^{4} \delta \\
& a_{10}=2\left(\frac{\pi R}{l}\right)^{2} \delta n^{2}, \quad a_{11}=1+\delta n^{4}-\Omega, \quad a_{12}=1+\delta n^{4} \\
& q_{1}=\frac{\pi R}{l} \frac{1-\nu}{2}\left(1-\frac{3}{4} \delta, n, \quad q_{2}=-a_{5}, \quad q_{3}=-\left(\frac{\pi R}{l}\right)^{2} \frac{3(1-\nu)}{2} \delta n\right. \\
& \left.q_{4}=-i \frac{\pi R}{l}\right) \frac{1-v}{2} \delta n^{2}, \quad q_{5}=-a_{7}, \quad q_{6}=-\left(a_{9} m^{2}+q_{12}\right) \\
& q_{12}=\frac{\pi R}{2}^{2}(2-v) \delta n^{2}, \quad q_{13}=q_{23} n \text {. } \\
& q_{18}=2\left(\frac{\pi R}{l}\right)^{2}(1-v) \delta n^{2}, \quad q_{23}=-\left(\frac{\pi R}{l}\right)^{2} v \delta n \\
& s_{11}=a_{1} k^{2}+a_{21}-\Omega, \quad s_{12}=a_{3} k, \quad s_{13}=a_{4} k \\
& s_{22}=a_{5} k^{2}+a_{61}-\Omega, \quad s_{23}=a_{7} k^{2}+a_{8}, \quad s_{33}=a_{9} k^{4}+a_{10} k^{2}+a_{12}-\Omega \\
& \left.a_{\alpha}=\left(s_{22} s_{33}-s_{23}^{2}\right) / \square\right]_{k n}, \quad a_{\beta}=\left(s_{13} s_{23}-s_{12} s_{33}\right) / L j_{k n} \\
& a_{\gamma}=\left(s_{12} s_{23}-s_{13} s_{22}\right) /\left[j_{k n}, \quad b_{\beta}=\left(s_{11} s_{33}-s_{13}^{2}\right) /\lfloor]_{k n}\right. \\
& b_{\gamma}=\left(s_{12} s_{13}-s_{11} s_{23}\right) /[]_{k n}, \quad c_{\gamma}=\left(s_{11} s_{22}-s_{12}^{2}\right) / \square_{k n} \\
& \square_{k n}=\left|\begin{array}{lll}
s_{11} & s_{12} & s_{13} \\
s_{12} & s_{22} & s_{23} \\
s_{13} & s_{23} & s_{33}
\end{array}\right|
\end{aligned}
$$


44

APPENDIX E

Elements of Frequency Determinant Using the First

Set (CSS) of Displacement Functions

$$
\begin{aligned}
& e_{1,1}=\frac{1}{2 a_{2}}+\sum_{k=1}^{\infty} a_{\alpha}, \quad e_{1,2}=\frac{1}{2 a_{2}}+\sum_{k=1}^{\infty} a_{\alpha}(-1)^{k}, \\
& e_{1,3}=\sum_{k=1}^{\infty} a_{\gamma}, \quad e_{1,4}=\sum_{k=1}^{\infty} a_{\gamma} k(-1)^{k}, \\
& e_{1,5}=\frac{q_{1}}{2 a_{2}}+\sum_{k=1}^{\infty}\left\{q_{1} a_{\alpha}+q_{2} a_{B} k+q_{3} a_{\gamma} k\right\} \\
& e_{1,6}=\frac{q_{1}}{2 a_{2}}+\sum_{k=1}^{\infty}\left\{q_{1} a_{\alpha}+q_{2} a_{\beta} k+q_{3} a_{\gamma} k\right\}(-1)^{k} \\
& e_{1,7}=\frac{q_{4}}{2 a_{2}}+\sum_{k=1}^{\infty}\left\{q_{4} a_{\alpha}+q_{5} a_{\beta} k+q_{6} a_{\gamma} k\right\} \\
& e_{1,8}=\frac{q_{4}}{2 a_{2}}+\sum_{k=1}^{\infty}\left\{q_{4} a_{\alpha}+q_{5} a_{\beta} k+q_{6}{ }^{a} k\right\}(-1)^{k} \\
& e_{2,2}=e_{1,1}, \quad e_{2,3}=\epsilon_{1,4}, \quad e_{2,4}=e_{1,3}, \quad e_{2,5}=e_{1,6}, \\
& e_{2,6}=e_{1,5}, e_{2,7}=e_{1,8}, e_{2,8}=e_{1,7} \\
& e_{3,3}=\sum_{k=1}^{\infty} c_{\gamma} k^{2}, \quad e_{3,4}=\sum_{k=1}^{\infty} c_{\gamma} k^{2}(-1)^{k}, \\
& e_{3,5}=\sum_{k=1}^{\infty}\left\{q_{1} c_{\alpha} k+q_{2} c_{B} k^{2}+q_{3} c_{\gamma}\right\} \\
& e_{3,6}=\sum_{k-1}^{\infty}\left\{q_{1} c_{\alpha} k+q_{2} c_{\beta} k^{2}+q_{3} c_{\gamma} k\right\}(-1)^{k} \\
& e_{3,7}=\frac{1}{2}+\sum_{k=1}^{\infty}\left\{q_{4} c_{\alpha} k+q_{5} c_{\beta} k^{2}+q_{6} c_{\gamma} k^{2}+1\right\}
\end{aligned}
$$


45

$e_{3,8}=\frac{1}{2}+\sum_{k=1}^{\infty}\left\{q_{4} c_{\alpha} k+q_{5} c_{\beta} k^{2}+q_{6} c_{\gamma} k^{2}+1\right\}(-1)^{k}$

$$
\begin{aligned}
& e_{4,4}=e_{3,3}, \quad e_{4,5}=e_{3,6}, \quad e_{4,6}=e_{3,5}, \quad e_{4,7}=e_{3,8}, \\
& e_{4,8}=e_{3,7}
\end{aligned}
$$

$$
\begin{aligned}
& e_{5,5}=\frac{q_{1}^{2}}{2 a_{2}}+\frac{q_{2}}{2}+\sum_{k=1}^{\infty}\left\{q_{1}^{2} a_{\alpha}+q_{2}^{2} b_{\beta} k^{2}+q_{3}^{2} c_{\gamma} k^{2}+2 q_{1} q_{2} a_{\beta} k\right. \\
& \left.+2 q_{2} q_{3} b_{\gamma} k^{2}+2 q_{1} q_{3} a_{\gamma} k+q_{2}\right\} \\
& e_{5,6}=\frac{q_{1}^{2}}{2 a_{2}}+\frac{q_{2}}{2}+\sum_{k=1}^{\infty}\left\{q_{1}^{2} a_{\alpha}+q_{2}^{2} b_{\beta} k^{2}+q_{3}^{2} c_{\gamma} k^{2}+2 q_{1} q_{2} a_{\beta} k\right. \\
& \left.+2 q_{2} q_{3} b_{\gamma} k^{2}+2 q_{1} q_{3}{ }^{a} k+q_{2}\right\}(-1)^{k} \\
& e_{5,7}=\frac{q_{1} q_{4}}{2 a_{2}}+\frac{q_{3}}{2}+\sum_{k=1}^{\infty}\left\{q_{4}\left(q_{1} a_{\alpha}+q_{2} b_{\alpha} k+q_{3} c_{\alpha} k\right)+q_{5}\left(q_{1} a_{\beta} k+q_{2} b_{\beta} k^{2}+q_{3} c_{\beta} k^{2}\right)\right. \\
& \left.+q_{6}\left(q_{1} a_{\gamma} k+q_{2} b k^{2}+q_{3} c_{\gamma} k^{2}\right)+q_{3}\right\} \\
& e_{5,8}=\frac{q_{1} q_{4}}{2 a_{2}}+\frac{q_{3}}{2}+\sum_{k=1}^{\infty}\left\{q_{4}\left(q_{1} a_{\alpha}+q_{2} b_{\alpha} k+q_{3} c_{\alpha} k\right)+q_{5}\left(q_{1} a_{\beta} k+q_{2} b_{\beta} k^{2}\right.\right. \\
& \left.\left.+q_{3} c_{\beta} k^{2}\right)+q_{6}\left(q_{1} a_{\gamma} k+q_{2} b_{\gamma} k^{2}+q_{3} c_{\gamma} k^{2}\right)+q_{3}\right\}(-1)^{k} \\
& e_{6,6}=e_{5,5}, e_{6,7}=e_{5,8}, \quad e_{6,8}=e_{5,7} \\
& e_{7,7}=\frac{q_{4}^{2}}{2 a_{2}}-\frac{q_{18}}{2}+\sum_{k=1}^{\infty}\left\{q_{4}\left(q_{4} a_{\alpha}+q_{5} b_{\alpha} k+q_{6} c_{\alpha} k\right)+q_{5}\left(q_{4} a_{B} k+q_{5} b_{B} k^{2}+q_{6} c_{B} k^{2}\right)\right. \\
& \left.+q_{6}\left(q_{4} a_{\gamma} k+q_{5} b_{\gamma} k^{2}+q_{6} c k^{2}\right)-a_{9} k^{2}-q_{18}\right\}
\end{aligned}
$$




$$
\begin{aligned}
\mathrm{e}_{7,8}= & \frac{q_{4}^{2}}{2 a_{2}}-\frac{q_{18}}{2}+\sum_{k=1}^{\infty}\left\{q_{4}\left(q_{4} a_{\alpha}+q_{5} b_{\alpha} k+q_{6} c_{\alpha} k\right)+q_{5}\left(q_{4} a_{\beta} k+q_{5} b_{\beta} k^{2}+q_{6} c_{\beta} k^{2}\right)\right. \\
& \left.+q_{6}\left(q_{4} a_{\gamma} k+q_{5} b_{\gamma} k^{2}+q_{6} c_{\gamma} k^{2}\right)-a_{9} k^{2}-q_{18}\right\}(-1)^{k} \\
e_{8,8}= & e_{7,7}
\end{aligned}
$$




\section{REFERENCE。;}

1. Arnold, R. N., and Warburton, G. B., "Flexural Vibrations of the Walls of Thin Cylindrical Shells Having Freely Supported Ends," Proc. Roy. Soc. London, Al97, 1948, pp. 238-256.

2. Arnold, R. N., and Warburton, G. B., "The Flexural Vibrations of Thin Cylinders," Proc. Inst. of Mech. Engrs., Al67, 1953, pp. 62-80.

3. Leissa, A. W., "Vibration of She11s," 1973, NASA SP-288.

4. Sharma, C. B. : and Johns, D. J., "Free Vibration of Cantilever Circular Cylindrical Shells - A Comparative Study," J. of Sound and Vibration, Vol. 25, No. 3, 1972, pp. 433-449.

5. Sharma, C. B., "Calculation of Natural Frequencies of Fixed-Free Circular Cylindrical Shells," J. of Sound and Vibration, Vol. 35, No. 1, 1974 , pp. 55-76.

6. Forsberg, K., "Influence of Boundary Conditions on the Modal Characteristics of Thin Cylindrical Shells," AIAA Journal, Vol. 2, No. 12, Dec. 1964, pp. 2150-2157.

7. Forsberg, K., "Axisymmetric ard Beam-Type Vibrations of Thin Cylindrical Shells," AIAA Journa1, Vo1. 7, No. 2, Feb. 1969, pp. 221-227.

8. Forsberg, K., "A Review of Analytical Methods used to Determine the Modal Characteristics of Cylindrical Shells," NASA CR-613, June 1965.

9. Warburton, G. B., "Vibration of Thin Cylindrical Shells," Journal of Mechanical Engineering Science, Vol. 7, No. 4, 1965, pp. 399-407.

10. Warburton, G. B., and Higgs, J., "Natural Frequencies of Thin Cantilever Cylindrical Shells," J. of Sound and Vibration, Vol. 11, No. 3, 1970, pp. $335-338$.

11. Goldman, R. L., "Mode Shapes and Frequencies of Clamped-Clamped Cylindrical Shells," AIAA Journa1, Vo1. 12, No. 12, Dec. 1974, pp. 1755-1756. 
12. Chung, Ho, "A General Miethod of Solution for Vibrations of Cylindrical Shells," Ph.D. Thesis, Tufts University (March 1974).

13. Sanders, J. L., Jr., "An Improved First Approximation Theory for Thin She1ls," Rept. 24, June 1959, NASA.

14. Budiansky, B., and Sanders, J. L., Jr., "On the Best First-order Linear Shell Theory," 1963 Progress in Applied Mlechanics - The Prager Anniversary Volume, $1963,129-140$.

15. Bromwich, T. J. I'a, "An Introduction to the Theory of Infinite Series," 2nd Edition Rev., Mackillan, London, 1955.

16. Kraus, H., Thin Elastic Shells, John Wiley \& Sons, Inc., 1967.

17. Budiansky, B., and Di Prima, R. C., "Bending Vibrations of Uniform Twisted Beams," J. of Mathematics and Physics, Vol. 39, No. 4, December 1960 , Pp. $237-245$.

18. Dym, C. L., "Some New Results for the Vibrations of Circular Cylinders," J. of Sound and Vibration, Vol. 29, No. 2, 1973, pp. 189-205.

19. Jolley, L. B. W., Sumation of Series, 2nd Edition Rev., Dever, New York, 1961.

20. Mulcahy, T. M., Turula, P., Chung, H., and Jendrzejczyk, .T. A., "Analytical. and Experimental Study of Two Concentric Cylinders Coupled by a Fluid Gap," Technica1 Memorandum ANL-CT-75-36, Argonne Nationa1 Laboratory, April 1975.

21. Bathe, K-J., Wilson, E. L., and Peterson, F. E., "SAP IV - A Structural Analysis Program for Static and Dynamic Response of Linear Systems," National Technical Information Service, EERC 73-11, June 1973.

22. McCormick, C. W., The NASTRAN User's Manual (level 15), National Aeronautics and Space Administration (1972). 